\title{
Comparative Analysis of Nitrides Band Structures Calculated by the Empirical Pseudopotential Method
}

\author{
Pierre Ziade $^{1, *}$, Christophe Palermo ${ }^{2}$, Antonio Khoury ${ }^{1}$, Roland Habchi $^{1}$, \\ Myriam Rahal ${ }^{3}$, Luca Varani ${ }^{2}$ \\ ${ }^{1}$ Research platform of nanosciences and nanotechnologies, Pierre Gemayel campus, Fanar, \\ Lebanese University, 90239 Lebanon \\ ${ }^{2}$ Institut d'Électronique du Sud (CNRS UMR 5214), Université Montpellier II, France \\ ${ }^{3}$ Faculté des Sciences II, Université Libanaise, Fanar, Lebanon \\ *Corresponding Author: ziade@ies.univ-montp2.fr
}

Copyright (C2014 Horizon Research Publishing All rights reserved.

\begin{abstract}
The electronic band structures of zinc blende and wurtzite $\mathrm{GaN}$ and $\mathrm{InN}$ are calculated using the empirical pseudopotential method, with the form factors adjusted to reproduce correctly the most important band features. To this end, a comprehensive analysis and comparison with several experimental and theoretical data reported in the literature is performed. Relevant energy spacings as well as direct and indirect band gaps are then derived from the band structures. The electron effective masses at high symmetry points are also obtained using a parabolic line fit. The calculated parameters are reported together with existing data so that they can be easily compared and used in the interpretation of experiments and for numerical simulation purposes.
\end{abstract}

Keywords Nitrides, Band structure, Pseudopotential empirical method

\section{Introduction}

In recent years, the interest in III-V nitrides has been increasing considerably because of their important technological applications due to their interesting electrical and optical properties. In particular, they have been widely investigated and exploited in the development of optoelectronic devices in the blue and ultraviolet spectral regions as well as for high power and high temperature electronics $[1,2,3,4,5,6,7,8]$. An additional feature further enhances the III-V nitrides potential over other semiconductors: the strong interaction between the electrons and optical phonons [9, 10], a crucial property for the development of terahertz devices. In this context, it is very important to have access to all the information about the band structure details and transport parameters that are necessary in both experimen- tal applications (device design and optimization), and theoretical modelling (Monte Carlo simulations, hydrodynamic calculations, etc. ) [11].

Binary nitrides such as GaN, AlN and InN usually cristallize in the wurtzite structure, but can also be grown in the zinc blende structure $[12,13,14]$. Early studies of the interband optical absorption performed on InN thin films were interpreted as being consistent with a fundamental energy gap of about $2 \mathrm{eV}$ $[15,16,17,18,19]$. Since 2002, improvements in epitaxial growth techniques enabled the availability of both wurtzite and zinc blende InN films of much higher quality with considerably low electron concentrations, much higher electron mobilities, but also lower oxygen defects caused by annealing in an oxygen atmosphere that leads to formation of $\mathrm{InN}-\mathrm{InO}_{2}$ alloys. Characterization of the obtained high-quality InN layers revealed for InN a band gap lower than $1 \mathrm{eV}[20,21,22]$, much smaller than the previously reported values. Parallel to the experimental improvements, theoretical band structure calculations of wurtzite and zinc blende $\mathrm{InN}$ are being revised in order to fit experimental results $[23,24]$. In this context, we present a theoretical investigation of the zinc blende and wurtzite band structures using an empirical pseudopotential approach (EPM), while providing sets of adjusted symmetrical and antisymmetrical pseudopotential form factors for InN consistently with those latest experimental results, but also for GaN and AlN whose band structures are also calculated for both zinc blende and wurtzite structures, in order to further validate our calculations. This will help the understanding of nitrides electronic properties and their important band structure parameters such as direct and indirect band gaps, effective masses and nonparabolicity coefficients.

The paper is organized as follows. The EPM is described in details for the zinc blende and wurtzite structures in Sections 2 and 3 respectively, in addition to the calculation of the nitrides band structures and derivation of the main energy spacings and effective masses. Sec- 
tion 4 summarizes the results, while presenting a comparative analysis of the main III-nitrides parameters.

\section{Band structure of zinc blende nitrides}

\subsection{Zinc blende structure}

Let consider in the left panel of Fig. 1 the facecentered cubic lattice whose base vectors are given by: $\mathbf{a}_{1}=(0, a / 2, a / 2), \mathbf{a}_{2}=(a / 2,0, a / 2)$, and $\mathbf{a}_{3}=$ $(a / 2, a / 2,0)$, where $a$ is the side of the lattice [25]. The zinc blende structure is formed from this face-centered cubic lattice by placing at each site of the lattice an elementary pattern composed of an atom of zinc and an atom of sulphur separated by a distance equal to one quarter of the diagonal of the unit cell (see Fig. 1).
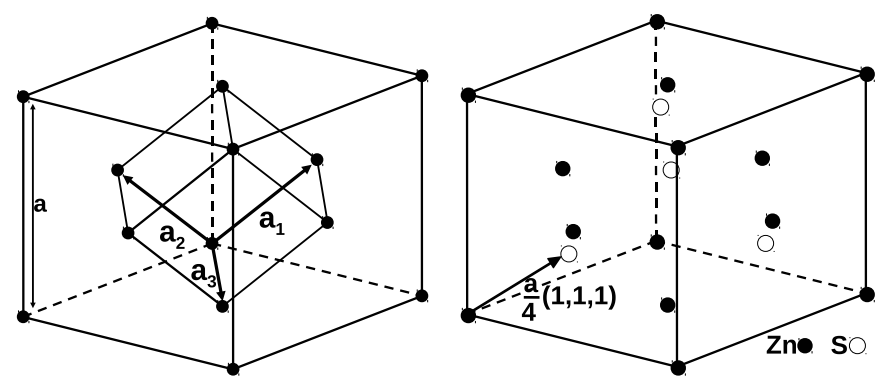

Figure 1. Face-centered cubic structure (left) and zinc blende lattice (right).

The reciprocal lattice of a face-centered cubic lattice, and as a consequence for a zinc blende structure, is a centered cubic lattice whose base vectors are given by $[25]: \quad \mathbf{b}_{\mathbf{1}}=(2 \pi / a)(-1,1,1)$, $\mathbf{b}_{\mathbf{2}}=(2 \pi / a)(1,-1,1)$, and $\mathbf{b}_{\mathbf{3}}=(2 \pi / a)$ $(1,1,-1)$. Given the high symmetry of the Brillouin zone, the band structure calculations can be considerably reduced using the symmetry operations of the crystal. It can be easily proved that a reduced region representing $1 / 48^{t h}$ of the Brillouin zone is sufficient to determine the energetic structure of the zinc blende crystals [25]. The reduced region is delimited by the following points whose coordinates are given in units of $(2 \pi / a): \Gamma=(0,0,0), X=(0,1,0), K=(0.75,0.75,0)$, $L=(0.5,0.5,0.5)$, and $U=(0.25,1,0.25)$ (see left panel of Fig. 2).
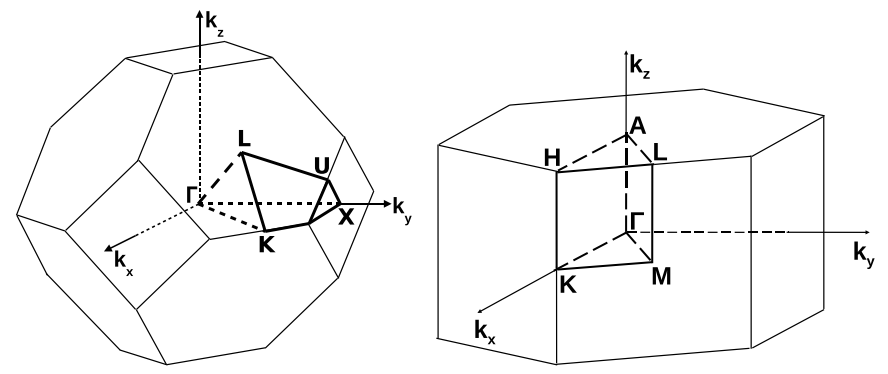

Figure 2. Brillouin zones of the zinc blende (left) and wurtzite (right) structures.

\subsection{Semi-empirical pseudopotential calcula- tion for zinc blende nitrides}

The one-electron Schrödinger equation resulting from the variational method by using a base of plane waves is $[26]$ :

$$
\left[\frac{\hbar^{2}}{2 m}(\mathbf{k}+\mathbf{g})^{2}-\epsilon\right] C_{\mathbf{k}+\mathbf{g}}+\sum_{\mathbf{g}^{\prime}} V_{\mathbf{g}-\mathbf{g}^{\prime}} C_{\mathbf{k}+\mathbf{g}^{\prime}}=0
$$

where $\mathbf{k}$ is a vector of the first Brillouin zone, $\mathbf{g}$ a vector of the reciprocal lattice, $\epsilon$ the energy eigenvalues, $V_{\mathbf{g}-\mathbf{g}^{\prime}}$ $\left(V_{\boldsymbol{\Delta} \mathbf{g}}\right)$ the pseudopotential and $C$ the eigenvector of the Hamiltonian.

We notice that (1) can be reduced to the form $H C=$ $\epsilon C$, where the elements of the matrix $H$ can be written as:

$$
H_{\mathbf{g}, \mathbf{g}^{\prime}}=\left(\frac{\hbar^{2}}{2 m}\right)|\mathbf{k}+\mathbf{g}|^{2} \delta_{\mathbf{g}, \mathbf{g}^{\prime}}+V_{\mathbf{g}, \mathbf{g}^{\prime}}
$$

In this expression, $V_{\mathbf{g}, \mathbf{g}^{\prime}}=V_{\boldsymbol{\Delta} \mathbf{g}}=\Sigma V_{r} \exp (-i \boldsymbol{\Delta} \mathbf{g} \cdot \mathbf{r})$, where the sum is over the atoms of the unit cell. We consider the origin at the midpoint between the two atoms of the unit cell so that $\mathbf{r}_{\mathbf{a}}=\left(\frac{a}{8}\right)(1,1,1)=\mathbf{s}$ and $\mathbf{r}_{\mathbf{b}}=\left(-\frac{a}{8}\right)(1,1,1)=-\mathbf{s}$ (see left panel of figure 3$)$.

$$
\begin{aligned}
V_{\Delta \mathrm{g}}= & V_{\Delta \mathrm{g}}^{s} \cos \left[\left(\frac{\pi}{4}\right)\left(\Delta g_{x}+\Delta g_{y}+\Delta g_{z}\right)\right] \\
& -i V_{\Delta \mathrm{g}}^{a} \sin \left[\left(\frac{\pi}{4}\right)\left(\Delta g_{x}+\Delta g_{y}+\Delta g_{z}\right)\right]
\end{aligned}
$$

where $V_{\Delta \mathrm{g}}^{s}$ and $V_{\Delta \mathrm{g}}^{a}$ are the symmetrical and antisymmetrical pseudopotential form factors respectively. They are treated as adjustable parameters that can be adapted with experimental data, which explains why the method is called semi-empirical pseudopotential method [26]. In other terms, the band structure calculations are made using experimental data that are adjusted until the results of the calculations agree with the experience.

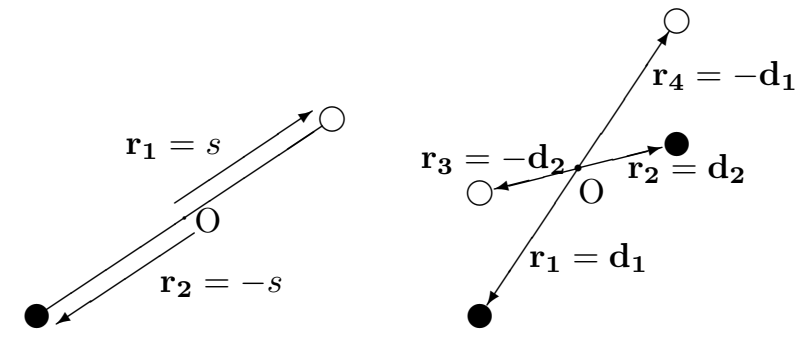

Figure 3. Elementary patterns of the zinc blende (left) and wurtzite (right) structures.

A vector $\mathbf{g}=l \mathbf{b}_{\mathbf{1}}+m \mathbf{b}_{\mathbf{2}}+n \mathbf{b}_{\mathbf{3}}$ of the reciprocal lattice can be expressed as:

$\mathbf{g}=\left(\frac{2 \pi}{a}\right)\left[(-l+m+n) \mathbf{a}_{\mathbf{1}}+(l-m+n) \mathbf{a}_{\mathbf{2}}+(l+m-n) \mathbf{a}_{\mathbf{3}}\right]$

Its norm is then given by:

$|\mathbf{g}|^{2}=\left(\frac{2 \pi}{a}\right)^{2}\left(3 l^{2}+3 m^{2}+3 n^{2}-2 l m-2 m n-2 l n\right)$

Therefore, the groups of vectors of the zinc blende structure are (coordinates are in units of $2 \pi / a$ ):

- $\mathbf{g}_{\mathbf{0}} \equiv(0,0,0)$ (the origin) 
60 Comparative Analysis of Nitrides Band Structures Calculated by the Empirical Pseudopotential Method

Table 1. Pseudopotential form factors (in Ry) of zinc blende GaN, AlN and InN used in the calculations.

\begin{tabular}{lllllll}
\hline \hline & $\mathbf{V}_{\mathbf{3}}^{\mathbf{s}}$ & $\mathbf{V}_{\mathbf{8}}^{\mathbf{s}}$ & $\mathbf{V}_{\mathbf{1 1}}^{\mathbf{s}}$ & $\mathbf{V}_{\mathbf{3}}^{\mathbf{a}}$ & $\mathbf{V}_{\mathbf{4}}^{\mathbf{a}}$ & $\mathbf{V}_{\mathbf{1 1}}^{\mathbf{a}}$ \\
\hline GaN & -0.2218 & 0.0180 & 0.0989 & 0.3360 & 0.2430 & 0.0400 \\
\hline AlN & -0.3284 & -0.0374 & 0.2341 & 0.2311 & 0.2757 & -0.0101 \\
\hline InN & -0.2186 & -0.0055 & 0.0569 & 0.0811 & 0.0531 & 0.0314 \\
\hline \hline
\end{tabular}

Table 2. Eigenenergies of zinc blende GaN, AlN and InN for the high-symmetry points $\Gamma, X$ and $L$. The values are in eV and are given with respect to $\Gamma_{15}^{v}$. Our calculations are evidenced by the asterisk, while all other values have been taken from the literature.

\begin{tabular}{|c|c|c|c|}
\hline & $\mathrm{aN}$ & $\mathrm{AlN}$ & $\operatorname{InN}$ \\
\hline$\Gamma_{15}^{\mathrm{V}}$ & $0.00^{*}, 0^{[27]}, 0.0(0.0)^{[28]}, 0.000^{[18,29,30]}$ & $0.00^{*}, 0^{[27]}, 0.000^{[18,29]}, 0.0(0.0)^{\lfloor 28]}$ & $0.00^{*}, 0.0^{[24]}, 0.000^{[23,31]}$ \\
\hline$\Gamma_{1}^{c}$ & $\begin{array}{l}3.23^{*}, \quad 3.20^{[27]}, \quad 2.1(3.1)^{[28]}, \quad 3.1^{[32]} \\
3.200^{[33]}, 3.308^{[18]}, 3.383^{[29]}, 3.213^{[30]}\end{array}$ & $\begin{array}{l}5.88^{*}, \quad 5.91^{[27]}, \quad 5.914^{[33]}, \quad 6.0^{[32]}, \\
5.840^{[18]}, 5.936^{[29]}, 4.2(6.0)^{[28]}\end{array}$ & $0.51^{*}, 0.592^{[23]}, 0.435^{[31]}$ \\
\hline$\Gamma_{15}^{\mathrm{c}}$ & $\begin{array}{l}12.11^{*}, 12.14^{[27]}, 10.098^{[18]}, 10.300^{[29]} \\
10.248^{[30]}, 10.6(12.2)^{[28]}\end{array}$ & $\begin{array}{lll}13.99^{*}, & 14.16^{[27]}, & 12.579^{[18]}, \\
13.406^{[29]}, 12.3(14.6)^{[28]} & \end{array}$ & $9.08^{*}, 9.597^{[23]}, 10$ \\
\hline $\mathbf{X}_{3}^{\mathbf{v}}$ & $\begin{array}{lrr}-5.45^{*}, & -5.47^{[27]}, & -6.294^{[18]} \\
-6.149^{[29]}, & -5.923^{[30]},-6.5(-6.9)^{[28]}\end{array}$ & $\begin{array}{l}-5.19^{*}, \quad-5.26^{[27]}, \quad-5.388^{[18]}, \\
-5.262^{[29]},-5.0(-5.6)^{[28]}\end{array}$ & $\begin{array}{l}-5.41^{*}, \quad-5.5^{[24]}, \\
-4.795^{[23]},-4.687^{[31]}\end{array}$ \\
\hline $\mathrm{X}_{5}^{\mathrm{v}}$ & $\begin{array}{l}-2.28^{*}, \quad-2.29^{[27]}, \quad-2.459^{[18]}, \\
-2.693^{[29]},-2.086^{[30]},-2.8(-3.0)^{[28]}\end{array}$ & $\begin{array}{l}-1.92^{*}, \quad-1.98^{[27]}, \quad-2.315^{[18]}, \\
-2.337^{[29]},-1.8(-2.1)^{[28]}\end{array}$ & $\begin{array}{l}-2.25^{*}, \quad-2.8^{[24]} \\
-1.481^{[23]},-1.687^{[31]}\end{array}$ \\
\hline $\mathrm{X}_{1}^{\mathrm{c}}$ & $\begin{array}{l}4.72^{*}, \quad 4.70^{[27]}, \quad 4.428^{[18]}, \quad 4.571^{[29]} \\
4.585^{[30]}, 4.7^{[32]}, 4.700^{[33]}, 3.2(4.7)^{[28]}\end{array}$ & $\begin{array}{l}4.91^{*}, \quad 4.92^{[27]}, \quad 4.918^{[33]}, \quad 4.9^{[32]}, \\
5.346^{[18]}, 5.102^{[29]}, 3.2(4.9)^{[28]}\end{array}$ & $4.38^{*}, 4.758^{[23]}, 2.903^{[31]}$ \\
\hline $\mathbf{X}_{3}^{\mathbf{c}}$ & $\begin{array}{l}7.45^{*}, \quad 7.46^{[27]}, \quad 6.010^{[18]}, \\
6.265^{[30]}, 12.2(14.5)^{[28]}\end{array}$ & $\begin{array}{l}10.07^{*}, 10.10^{[27]}, 8.794^{[18]}, 10.661^{[29]}, \\
14.1(17.3)^{[28]}\end{array}$ & $5.78^{*}, 5.102^{[23]}, 6.322$ \\
\hline $\mathbf{L}_{1}^{\mathbf{v}}$ & $\begin{array}{lrr}-6.15^{*}, & -6.19^{[27]}, & -6.812^{[18]} \\
-6.743^{[29]}, & -6.644^{[30]},-7.4(-7.8)^{[28]}\end{array}$ & $\begin{array}{l}-5.86^{*}, \quad-5.98^{[27]}, \quad-6.251^{[18]}, \\
-6.179^{[29]},-6.0(-6.7)^{[28]}\end{array}$ & $\begin{array}{l}-6.51^{*}, \quad-6.5^{[24]}, \\
-4.967^{[23]},-5.229^{[31]}\end{array}$ \\
\hline $\mathbf{L}_{3}^{\mathbf{v}}$ & 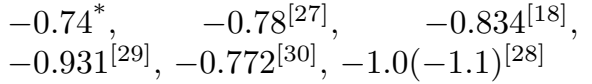 & $\begin{array}{l}-0.68^{*}, \quad-0.75^{[27]}, \quad-0.718^{[18]}, \\
-0.728^{[29]},-0.5(-0.6)^{[28]}\end{array}$ & $\begin{array}{l}-0.69^{*}, \quad-1.0^{[24]} \\
-0.460^{[23]},-0.557^{[31]}\end{array}$ \\
\hline $\mathbf{L}_{1}^{\mathrm{c}}$ & $\begin{array}{l}6.23^{*}, 6.20^{[27,32]}, 6.200^{[33]}, 5.149^{[18]} \\
5.636^{[29]}, 5.510^{[30]}, 5.0(6.2)^{[28]}\end{array}$ & $\begin{array}{l}9.14^{*}, \quad 9.08^{[27]}, \quad 9.079^{[33]}, \quad 9.3^{[32]}, \\
8.264^{[18]}, 9.423^{[29]}, 10.0(12.6)^{[28]}\end{array}$ & $4.39^{*}, 3.213^{[23]}, 3.813^{[31]}$ \\
\hline $\mathbf{L}_{3}^{\mathbf{c}}$ & $\begin{array}{l}11.22^{*}, 11.19^{[27]}, 10.416^{[18]}, 9.916^{[29]} \\
10.606^{[30]}, 10.6(12.3)^{[28]}\end{array}$ & $\begin{array}{lll}11.85^{*}, & 11.91^{[27]}, & 12.202^{[18]}, \\
12.014^{[29]}, 11.0(13.2)^{[28]} & \end{array}$ & $7.52^{*}, 10.040^{[23]}, 6.954^{[31]}$ \\
\hline
\end{tabular}

Table 3. Energy gaps of zinc blende GaN, AlN and InN for the high-symmetry points $\Gamma, X$ and $L$. Our calculations are evidenced by the asterisk, while all other values have been taken from the literature. All values are in $\mathrm{eV}$.

\begin{tabular}{|c|c|c|c|c|}
\hline & GaN & AlN & InN & \\
\hline $\mathrm{E}_{\mathrm{g}}^{\Gamma-\Gamma}$ & $\begin{array}{lll}3.23^{*}, 3.20^{[27,34]}, 3.10^{[35,28]}, & 3.21^{[30]}, \\
3.38^{[29,36]}, \quad 3.17^{[15]}, 3.82^{[37]}, & 3.26^{[38]}, \\
3.25^{[39]}, 3.43^{[40]}, 3.2^{[41]}, 3.299^{[42]}, & 2.08^{[43]}, \\
2.65^{[44]}\end{array}$ & 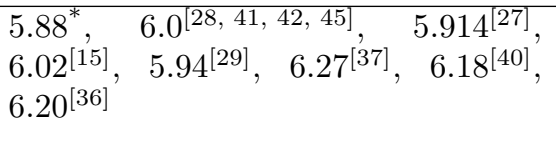 & $\begin{array}{l}0.51^{*} \\
0.435^{[31]}\end{array}$ & $0.592^{[23]}$ \\
\hline $\mathbf{E}_{\mathrm{g}}^{\Gamma-\mathbf{X}}$ & $\begin{array}{l}4.72^{*}, \quad 4.70^{[27,28,35]}, \quad 4.58^{[30]}, \\
4.57^{[29,40,36]}, 4.61^{[37]}, 4.7^{[41]}, 4.52^{[42]}\end{array}$ & $\begin{array}{l}4.91^{*}, \quad 4.9^{[28,41,42,45]}, \quad 4.918^{[27]}, \\
4.92^{[15]}, 5.10^{[29,40,36]}, 5.24^{[37]}\end{array}$ & $\begin{array}{l}4.38^{*} \\
2.903^{[31]}\end{array}$ & $4.758^{[23]}$ \\
\hline $\mathbf{E}_{\mathrm{g}}^{\Gamma-\mathbf{L}}$ & $\begin{array}{l}6.23^{*}, 6.20^{[27,28,35]}, 5.51^{[30]}, 5.64^{[29,36]} \\
5.97^{[37]}, 5.66^{[40]}, 6.2^{[41]}, 5.59^{[42]}\end{array}$ & $\begin{array}{l}9.14^{*}, 9.079^{[27]}, 9.3^{[28,41,42]}, 9.70^{[37]} \\
9.42^{[29,36]}, 9.43^{[40]}, 9.21^{[45]}\end{array}$ & $\begin{array}{l}4.39^{*} \\
3.831^{[31]}\end{array}$ & $3.213^{[23]}$, \\
\hline $\mathbf{E}_{\mathrm{g}}^{\mathrm{L}-\mathbf{L}}$ & $6.97^{*}, 6.9^{[38]}, 7.0^{[46]}$ & $9.82^{*}$ & $\begin{array}{l}5.08^{*} \\
3.85^{[31]}\end{array}$ & $3.673^{[23]}$, \\
\hline $\mathbf{E}_{\mathrm{g}}^{\mathbf{X}-\mathbf{X}}$ & $7.00^{*}, 7.2^{[38]}, 7.6^{[46]}$ & $6.83^{*}$ & $\begin{array}{l}6.63^{*}, \\
4.59^{[31]}\end{array}$ & $6.239^{[23]}$ \\
\hline
\end{tabular}


Table 4. Effective masses of zinc blende GaN, AlN and InN. Our calculations are evidenced by the asterisk, while all other values have been taken from the literature. All values are in units of the free electron mass.

\begin{tabular}{|c|c|c|c|c|}
\hline & 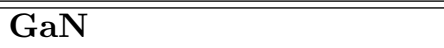 & & AlN & InN \\
\hline $\mathbf{m}_{\mathbf{e}}$ & $\begin{array}{lcc}0.1393^{*}, & 0.12^{[30]}, & 0.11^{[15]}, \\
0.15^{[47]}, & 0.14^{[38]}, & 0.21^{[48]}, \\
0.127^{[41]}, & (0.15 \pm 0.01)^{[39]}\end{array}$ & $\begin{array}{r}0.13^{[29]}, \\
0.124^{[49]},\end{array}$ & 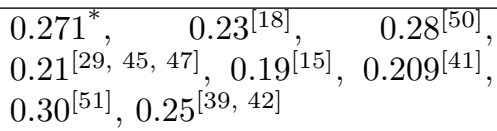 & $0.1140^{*}, 0.066^{[23]}, 0.07^{[52]}$ \\
\hline $\mathbf{m}_{\mathrm{hh}}^{[100]}$ & $\begin{array}{l}0.9807^{*}, \quad 0.84^{[18]}, \quad 0.86^{[50]}, \\
0.85^{[51]}, 0.85^{[42]}, 1.34^{[30]}\end{array}$ & $0.76^{[53]}$ & $\begin{array}{l}1.3114^{*}, \quad 1.02^{[18]}, \quad 1.44^{[50]}, \\
1.05^{[53]}, 1.39^{[51]}, 1.02^{[42]}\end{array}$ & $0.8381^{*}, 1.262^{[23]}, 0.83^{[52]}$ \\
\hline $\mathbf{m}_{\mathrm{lh}}^{[\mathbf{1 0 0}]}$ & $\begin{array}{l}0.1624^{*}, \quad 0.22^{[18]}, \quad 0.21^{[50]}, \\
0.24^{[51]}, 0.24^{[42]}, 0.70^{[30]}\end{array}$ & $0.21^{[53]}$ & $\begin{array}{l}0.2925^{*}, \quad 0.37^{[18]}, \quad 0.42^{[50]}, \\
0.35^{[53]}, 0.44^{[51]}, 0.35^{[42]}\end{array}$ & $0.0984^{*}, 0.100^{[23]}, 0.16^{[52]}$ \\
\hline $\mathbf{m}_{\mathrm{hh}}^{[111]}$ & $\begin{array}{l}2.6063^{*}, \quad 2.07^{[18]}, \quad 2.09^{[50]}, \\
1.79^{[51]}, 2.13^{[42]}, 1.06^{[30]}\end{array}$ & $1.93^{[53]}$ & $\begin{array}{l}3.0688^{*}, \quad 2.64^{[18]}, \quad 4.24^{[50]}, \\
2.73^{[53]}, 3.85^{[51]}, 4.55^{[42]}\end{array}$ & $2.2593^{*}, 2.744^{[23]}, 0.83^{[52]}$ \\
\hline $\mathbf{m}_{1 \mathrm{~h}}^{[111]}$ & $\begin{array}{l}0.1350^{*}, \quad 0.19^{[18]}, \quad 0.19^{[50]}, \\
0.21^{[51]}, 0.21^{[42]}, 0.63^{[30]}\end{array}$ & $0.18^{[53]}$ & $\begin{array}{l}0.2343^{*}, \quad 0.30^{[18]}, \quad 0.36^{[50]}, \\
0.30^{[53]}, 0.36^{[51]}, 0.28^{[42]}\end{array}$ & $0.0859^{*}, 0.096^{[23]}, 0.16^{[52]}$ \\
\hline $\mathbf{m}_{\mathrm{hh}}^{[110]}$ & $\begin{array}{l}0.9763^{*}, \quad 1.52^{[18]}, \quad 1.65^{[50]}, \\
1.40^{[51]}, 1.55^{[42]}, 1.44^{[30]}\end{array}$ & $1.51^{[53]}$ & $\begin{array}{l}1.3140^{*}, \quad 1.89^{[18]}, \\
2.16^{[53]}, 2.67^{[51]}, 2.44^{[42]}\end{array}$ & $0.8397^{*}, 2.122^{[23]}, 1.55^{[52]}$ \\
\hline $\mathbf{m}_{\mathrm{lh}}^{[110]}$ & $\begin{array}{l}0.1412^{*}, \quad 0.20^{[18]}, \quad 0.19^{[50]} \\
0.21^{[51]}, 0.21^{[42]}, 0.58^{[30]}\end{array}$ & $0.19^{[53]}$ & $\begin{array}{l}0.2468^{*}, \quad 0.32^{[18]}, \quad 0.37^{[50]}, \\
0.31^{[53]}, 0.38^{[51]}, 0.29^{[42]}\end{array}$ & $0.0887^{*}, 0.097^{[23]}, 0.15^{[52]}$ \\
\hline
\end{tabular}

- $\mathbf{g}_{3} \equiv(-1,-1,-1),( \pm 1,0,0),(0, \pm 1,0),(0,0, \pm 1)$, $(1,1,1)$

- $\mathbf{g}_{4} \equiv(-1,-1,0),(-1,0,-1),(0,-1,-1),(0,1,1)$, $(1,0,1),(1,1,0)$

- $\mathbf{g}_{\mathbf{8}} \equiv(-2,-1,-1),(-1,-2,-1), \quad(-1,-1,-2)$, $(-1,0,1),(-1,1,0),(0,-1,1),(0,1,-1),(1,-1,0)$, बे $(1,0,-1),(1,1,2),(1,2,1),(2,1,1)$

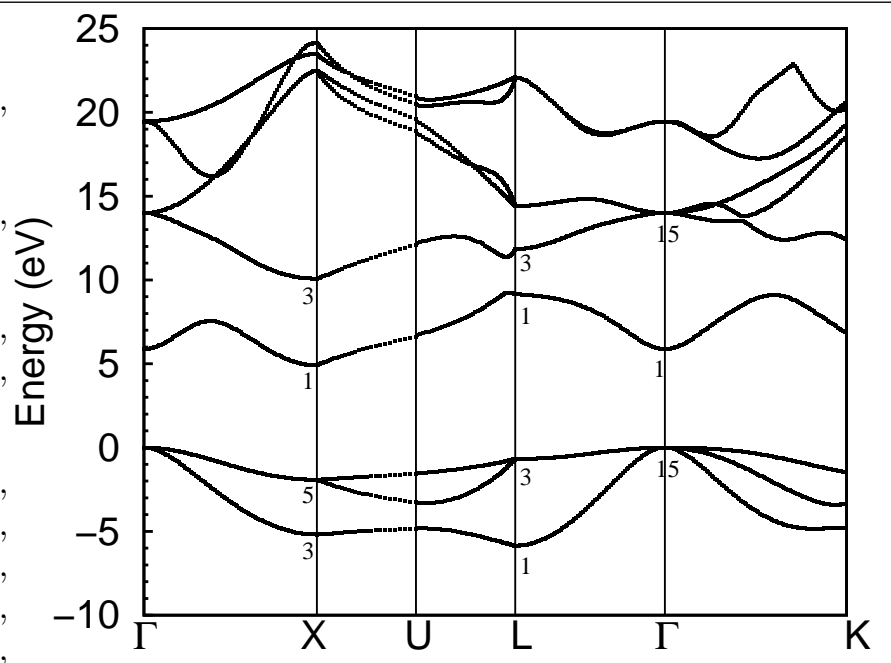

- $\mathbf{g}_{1 \mathbf{1}} \equiv(-2,-2,-1), \quad(-2,-1,-2), \quad(-2,-1,0)$, $(-2,0,-1),(-1,-2,-2),(-1,-2,0),(-1,-1,1)$, $(-1,0,-2), \quad(-1,1,-1), \quad(-1,1,1), \quad(0,-2,-1)$, $(0,-1,-2),(0,1,2),(0,2,1),(1,-1,-1),(1,-1,1)$ $(1,0,2), \quad(1,1,-1), \quad(1,2,0), \quad(1,2,2), \quad(2,0,1)$ $(2,1,0),(2,1,2),(2,2,1)$

- $\mathbf{g}_{\mathbf{1 2}} \equiv(-2,-2,-2),( \pm 2,0,0),(0, \pm 2,0),(0,0, \pm 2)$, $(2,2,2) \ldots$

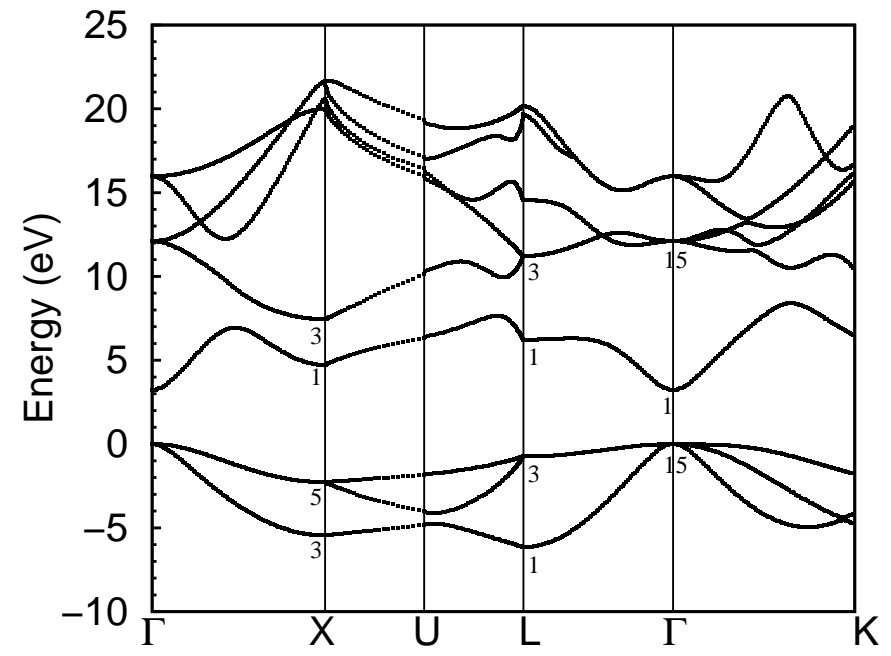

Figure 5. Band structure of zinc blende AlN obtained by our pseudopotential calculations.

value zero or any other adequate value. Besides, we can neglect the pseudopotential form factors corresponding to $\mathbf{g}^{2}>11(2 \pi / a)^{2}$, because $V_{\mathbf{g}}$ typically decreases as $1 / \mathbf{g}^{2}$ for high values of $g[26]$. Furthermore, the symmetrical term corresponding to $\mathbf{g}_{4}$ and the antisymmetrical term corresponding to $\mathbf{g}_{8}$ are always null because $\cos \left(\mathbf{g}_{\mathbf{4}} \cdot \mathbf{s}\right)=\sin \left(\mathbf{g}_{\mathbf{8}} \cdot \mathbf{s}\right)=0$ for any $\mathbf{g}_{\mathbf{4}}$ or $\mathbf{g}_{\mathbf{8}}$ vector. Therefore, only 6 pseudopotential form factors are sufficient for the calculation: $V_{3}^{s}, V_{8}^{s}, V_{11}^{s}, V_{3}^{a}, V_{4}^{s}$ and $V_{11}^{a}$.

In Table 1 are given the pseudopotential form factors of the zinc blende GaN and InN that we recommend and that we used in our calculations. In particular, the pseudopotential form factors of InN have been adapted to reproduce a band structure in good agreement with the latest experimental results. The lattice constants used for zinc blende GaN, AlN and InN are: $0.44953 \mathrm{~nm}$ [27], $0.43752 \mathrm{~nm}$ [27] and $0.4986 \mathrm{~nm}[54,55]$ respectively.

pseudopotential calculations.

Actually, only 4 groups of vectors are practically necessary for the calculations [26]. As a matter of fact, the form factor $V_{0}$ corresponding to $\mathrm{g}_{0}$ is a constant potential that shifts the whole energetic scale of a quantity $V_{0}$. This is the reason why we can attribute to $V_{0}$ the

We still have to choose the size of the Hamiltonian matrix whose diagonal elements are given by:

$$
H_{i, i}=\left(\frac{\hbar^{2}}{2 m}\right)\left|\mathbf{k}+\mathbf{g}_{\mathbf{i}}\right|^{2}
$$


and non-diagonal elements are given by:

$$
\begin{aligned}
H_{i, j}= & V_{s}\left(\left|\mathbf{g}_{\mathbf{i}}-\mathbf{g}_{\mathbf{j}}\right|\right) \cos \left[\left(\mathbf{g}_{\mathbf{i}}-\mathbf{g}_{\mathbf{j}}\right) \cdot \mathbf{s}\right] \\
& +i V_{a}\left(\left|\mathbf{g}_{\mathbf{i}}-\mathbf{g}_{\mathbf{j}}\right|\right) \sin \left[\left(\mathbf{g}_{\mathbf{i}}-\mathbf{g}_{\mathbf{j}}\right) \cdot \mathbf{s}\right]
\end{aligned}
$$

for $i \neq j$.

It is evident that the size of the Hamiltonian matrix depends on the chosen number of reciprocal lattice vectors. For that purpose, we choose a maximum value to limit the norm of the chosen reciprocal vectors given by (5). In our case, we have verified that the condition $|\mathbf{g}|^{2}<21(2 \pi / a)^{2}$ (corresponding to a set of 113 reciprocal vectors) provides sufficiently accurate results. In this way, we can calculate all the elements of the Hamiltonian matrix using (6) and (7). The diagonalization of the Hamiltonian matrix gives directly the eigenvalues of the electronic states corresponding to the vector $\mathbf{k}$ of the Brillouin zone.

\section{$2.3 \quad$ Results}

The energetic band structures obtained by our pseudopotential calculations are represented in figures 4,5 and 6 for zinc blende GaN, AlN and $\mathrm{InN}$ respectively, along the high-symmetry points $\Gamma, X, U, L$, and $K$ of the Brillouin zone. We notice that the energy reference level matches the top of the valence band. From a qualitative point of view, the overall shape of the valence and conduction bands that we have obtained for zinc blende GaN and AlN is similar to that obtained by Fritsch et al. [18], Al-Douri et al. [37], Pugh et al. [15] and Aourag et al. [27, 33]. The same occurs for zinc blende InN whose overall shape of the valence and conduction bands is similar to the reexamined band structures of Fritsch et al. [23] and King et al. [24].

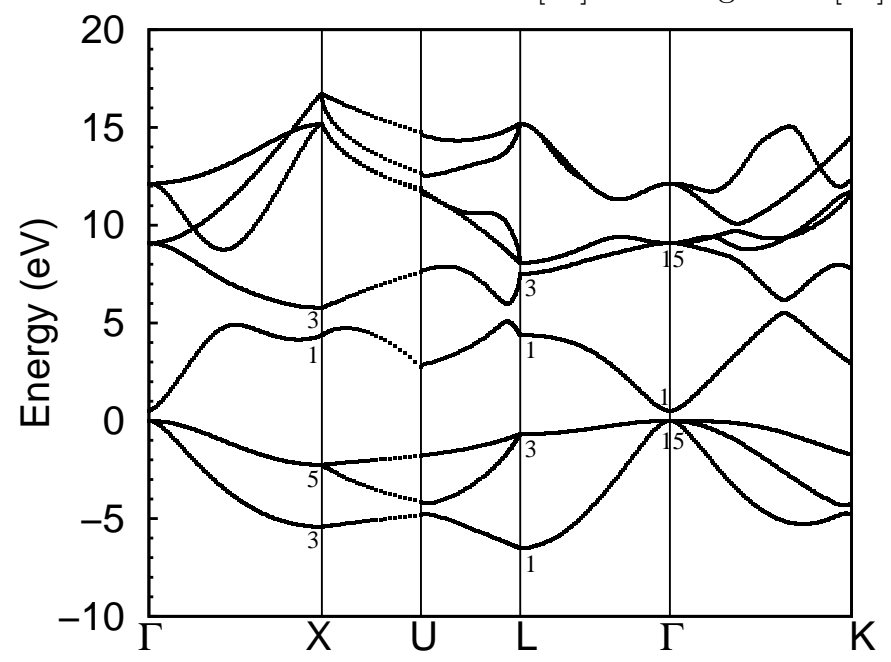

Figure 6. Band structure of zinc blende $\mathrm{InN}$ obtained by our pseudopotential calculations.

In order to quantitavely compare the obtained band structures with other results, the obtained energetic eigenvalues are given in Table 2 for the high-symmetry points $\Gamma, X$ and $L$. All eigenvalues are given with respect to the top of the valence band $\Gamma_{15}^{v}$. We note first that all results and calculations before 2002 that do not take into consideration the new corrections brought into the band structure of InN have not been included in our comparison. In addition, we note that the values corresponding to ref. [24] are approximate values that we have estimated from the band structure of zinc blende InN obtained by King et al. [24], since no accurate values are available in the article, but only the obtained band structure. On the other hand, in ref. [28], there are two values proposed for each energetic eigenvalue, both calculated by the local-density approximation (LDA), but the value in parenthesis is the result of an additional correction by the Green's function approximation (GW) which takes into consideration the Coulomb screening effect of the electrons [28]. We can initially point out the zero value assigned to $\Gamma_{15}^{v}$ by all cited references [27], [33], [32], [28], [18], [23], [29], and [30], which means that the energy reference level has been taken at the top of the valence band in all calculations. Regarding the results of zinc blende GaN and AlN, we remark an excellent agreement between our results and those found by Aourag et al. $[27,33]$ since the relative difference between the results does not exceed $2 \%$ for most of the energy eigenvalues, and even $0.5 \%$ for some of them.

We also observe a general agreement with the results of references [18], [29], [30] and [32] and ours, since their relative deviation does not exceed $10 \%$ for most energy eigenvalues. As for the calculations performed by Rubio et al. [28], there's a good agreement with our results for the eigenenergies $\Gamma_{15}^{v}, \Gamma_{1}^{v}, \Gamma_{15}^{c}, X_{5}^{v}, X_{1}^{c}$, and $L_{3}^{c}$ corresponding to GaN and AlN, for $L_{1}^{c}$ corresponding to GaN, and for $X_{3}^{v}$ and $L_{3}^{v}$ corresponding to AlN. But the eigenvalues $\Gamma_{1}^{v}, X_{1}^{v}, X_{3}^{c}$ of GaN and AlN are widely overestimated (by at least 20\%) in [28]. We also notice an underestimation of the eigenvalues $X_{3}^{v}$ and $L_{3}^{v}$ corresponding to $\mathrm{GaN}$ with respect to our results and to other calculations. On the other hand, regarding the results of zinc blende InN, we first remark a slight underestimation of most valence band eigenenergies obtained by our calculations with respect to those of references [23] and [31]. This is due to the fact that we have adapted our valence band structure to the very recent results by King et al. obtained by a combination of experimental characterization by high-resolution x-ray photoemission spectroscopy assisted by theoretical calculations via the quasiparticle corrected density functional theory [24]. Despite this slight underestimation, our values are generally in good agreement with the results of the cited works of Fritsch et al. [23], Bechstedt et al. [31] and of course King et al. [24].

In order to ensure the precision of our semi-empirical results in comparison to other semi-empirical and $a b$ initio calculations and experimental results, the values of the bandgaps $E_{g}^{\Gamma-\Gamma}, E_{g}^{\Gamma-X}, E_{g}^{\Gamma-L}, E_{g}^{X-X}$ and $E_{g}^{L-L}$ at high-symmetry points $\Gamma, X$ and $L$ are shown in Table 3. Regarding the energy spacings of GaN and AlN, we remark a good agreement between the values found by our calculations and those of most other references in Table 3. Even the bandgap values given by Rubio et al. [28] agree with our calculations. As a matter of fact, for most values of Table 3 , the relative difference between our calculations and those of the other references does not exceed $10 \%$. Only the values of $E_{g}^{\Gamma-\Gamma}$ proposed by Al-Douri et al. [37] and Palummo et al. [44] for zinc blende GaN make an exception: Palummo underestimates the gap value to $2.65 \mathrm{eV}$ and Al-Douri overestimates it to $3.82 \mathrm{eV}$. However, the value of $E_{g}^{\Gamma-\Gamma}$ for AlN and the values of $E_{g}^{\Gamma-X}$ and $E_{g}^{\Gamma-L}$ for GaN and 
AlN proposed by Al-Douri et al. are in good agreement with our calculations. The slight disagreements do not prevent a general good agreement with other theoretical and experimental results. As for the obtained energy spacings for zinc blende InN, they are in good agreement with the results of Fritsch et al. [23] and Bechstedt et al. [31], despite the slight overestimation of the energy spacings $E_{g}^{\Gamma-L}, E_{g}^{L-L}$ and $E_{g}^{X-X}$ due to the adaptation of our InN valence band structures with the very recent results and theoretical calculations performed by King et al. [24], as we have already explained.

On the other hand, the effective electron mass is a fundamental quantity of semiconductors, whose knowledge is necessary for the interpretation of several experiments and theoretical models. Its value at the bottom of the conduction band (or at the top of the valence band) can be obtained from the band structure of the material under investigation using the parabolic approximation

$$
\frac{1}{m^{*}}=\frac{4 \pi^{2}}{h^{2}} \frac{\partial^{2} E(k)}{\partial k^{2}}
$$

We have calculated through the parabolic approximation the electronic effective mass at $\Gamma$ point, but also the light hole (lh) and heavy hole (hh) effective masses along the $[1,0,0],[1,1,1]$ and $[1,1,0]$ directions for zinc blende GaN, AlN and InN. Our obtained results are given in Table 4 in comparison with other theoretical and experimental values available in the literature. Concerning the electronic effective masses, our results agree with most references cited in Table 4 . As for the hole effective masses, we remark a discrepancy between the values given by the different cited references, which prevents to assert definitely the validity of our results. Nevertheless, although we use the parabolic approximation method which is simple compared to more sophisticated methods, some of our values actually coincide with the results from other references.

\section{Band structure of wurtzite ni- trides}

\subsection{Wurtzite structure}

The base vectors of the hexagonal lattice are shown in the left panel of Fig. $7: \mathbf{a}_{\mathbf{1}}=(a, 0,0), \mathbf{a}_{2}=\left(\frac{a}{2}\right)(1, \sqrt{3}, 0)$ and $\mathbf{a}_{\mathbf{3}}=(0,0, c)[25]$. The compact hexagonal structure is made from the hexagonal lattice by placing at each site of the lattice two atoms separated by a vector $\mathbf{t}=\left(\frac{1}{3} \mathbf{a}_{1}+\frac{1}{3} \mathbf{a}_{2}+\frac{1}{2} \mathbf{a}_{3}\right)$ (see middle panel of figure 7). Finally, the wurtzite structure is made from the compact hexagonal lattice by placing at each site two atoms separated by a vector $u \mathbf{a}_{3}$, where $u \approx 0.375$ is a parameter relative to the wurtzite structure (right panel of figure 7). Therefore, as a result, the elementary pattern of the wurtzite structure is made of 4 atoms.

The reciprocal lattice of the hexagonal lattice is also hexagonal. The base vectors of this reciprocal lattice are given by: $\mathbf{b}_{\mathbf{1}}=\left(\frac{2 \pi}{a \sqrt{3}}\right)(\sqrt{3}, 1,0), \mathbf{b}_{\mathbf{2}}=\left(\frac{4 \pi}{a \sqrt{3}}\right)(0,1,0)$ and $\mathbf{b}_{\mathbf{3}}=\left(\frac{2 \pi}{c}\right)(0,0,1)$. Thus, the first Brillouin zone of the reciprocal lattice of the hexagonal structure is also hexagonal. Given the high symmetry of the Brillouin

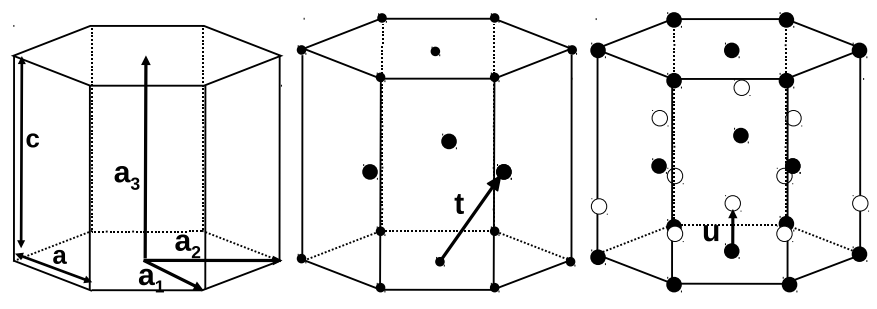

Figure 7. Hexagonal lattice (left), compact hexagonal structure (middle) and wurtzite structure (right).

zone, the band structure calculations can be considerably reduced using the symmetry operations of the crystal. In particular, it can easily be proved that a reduced region representing $1 / 24^{\text {th }}$ of the Brillouin zone such as reported in the right panel of Fig. 2 is sufficient to determine the energetic structure of the wurtzite crystals. This reduced region is delimited by the following points: $\Gamma=(0,0,0)$ (origin), $H=\left(\frac{2 \pi}{3 a}, \frac{2 \pi}{a}, \frac{2 \pi}{c}\right)$ (extremity of an edge joining two rectangular sides), $A=\left(0,0, \frac{\pi}{c}\right)$ (center of an hexagonal side), $K=\left(\frac{2 \pi}{3 a}, \frac{2 \pi}{a}, 0\right)$ (middle of an edge joining two rectangular sides $), L=\left(0, \frac{2 \pi}{a}, \frac{2 \pi}{c}\right)$ (middle of an edge joining an hexagonal and a rectangular side), and $M=\left(0, \frac{2 \pi}{a}, 0\right)$ (center of a rectangular side).

\subsection{Semi-empirical pseudopotential cal- culation for wurtzite nitrides}

The elementary pattern of the wurtzite structure is made of 4 atoms (see right panel of figure 3). By considering the origin at the center of the parallelogram formed by these atoms, their positions according to the base vectors $\left(\mathbf{a}_{1}, \mathbf{a}_{2}, \mathbf{a}_{3}\right)$ are given by: $\mathbf{r}_{\mathbf{1}}=\left(-\frac{1}{6}-\frac{1}{6},-\frac{1}{4}-\frac{u}{2}\right)$, $\mathbf{r}_{2}=\left(\frac{1}{6}, \frac{1}{6}, \frac{1}{4}-\frac{u}{2}\right), \mathbf{r}_{3}=\left(-\frac{1}{6},-\frac{1}{6},-\frac{1}{4}+\frac{u}{2}\right)$ and $\mathbf{r}_{\mathbf{4}}=$ $\left(\frac{1}{6}, \frac{1}{6}, \frac{1}{4}+\frac{u}{2}\right)$. Let $\mathbf{d}_{\mathbf{1}}=\mathbf{r}_{\mathbf{1}}=-\mathbf{r}_{\mathbf{4}}$ and $\mathbf{d}_{\mathbf{2}}=\mathbf{r}_{\mathbf{2}}=-\mathbf{r}_{\mathbf{3}}$. According to the unit vectors $(\mathbf{i}, \mathbf{j}, \mathbf{k})$, the expressions of $\mathbf{d}_{\mathbf{1}}$ and $\mathbf{d}_{\mathbf{2}}$ become: $\mathbf{d}_{\mathbf{1}}=\left(\frac{1}{4}, \frac{\sqrt{3}}{12}, \frac{1+2 u}{\sqrt{6}}\right) a$, and $\mathbf{d}_{\mathbf{2}}=$ $\left(\frac{1}{4}, \frac{\sqrt{3}}{12}, \frac{1-2 u}{\sqrt{6}}\right) a$.

Because the atoms at positions $\mathbf{r}_{1}$ and $\mathbf{r}_{2}$ are identical (same atomic pseudopotential $V_{1}$ ), and the atoms at positions $\mathbf{r}_{3}$ and $\mathbf{r}_{4}$ are also identical (same atomic pseudopotential $V_{2}$ ), the expression of the pseudopotential is given by: $V_{\boldsymbol{\Delta}}=V_{1}\left(\exp i \boldsymbol{\Delta} \mathbf{g} \cdot \mathbf{r}_{\mathbf{1}}+\exp i \boldsymbol{\Delta} \mathbf{g} \cdot \mathbf{r}_{\mathbf{2}}\right)+$ $V_{2}\left(\exp i \boldsymbol{\Delta} \mathbf{g} \cdot \mathbf{r}_{\mathbf{3}}+\exp i \boldsymbol{\Delta} \mathbf{g} \cdot r_{4}\right)$. The calculation leads to the final expression:

$$
V(|\boldsymbol{\Delta} \mathbf{g}|)=V^{s}(|\boldsymbol{\Delta} \mathbf{g}|) S^{s}(|\boldsymbol{\Delta} \mathbf{g}|)+i V^{a}(|\boldsymbol{\Delta} \mathbf{g}|) S^{a}(|\boldsymbol{\Delta} \mathbf{g}|)
$$

where $S^{s}$ and $S^{a}$ are the symmetrical and antisymmetrical structure factors with:

$$
S^{s}=\cos \left[2 \pi\left(\frac{\Delta l}{6}+\frac{\Delta m}{6}+\frac{\Delta n}{4}\right)\right] \cos (\pi u \Delta n)
$$

and

$$
S^{a}=\cos \left[2 \pi\left(\frac{\Delta l}{6}+\frac{\Delta m}{6}+\frac{\Delta n}{4}\right)\right] \sin (\pi u \Delta n)
$$

and where $V^{s}$ and $V^{a}$ are the symmetrical and antisymmetrical pseudopotential form factors.

A vector of the reciprocal lattice can be expressed as: $\mathbf{g}=\frac{2 \pi}{a}\left(l \mathbf{i}+\frac{2 m-l}{\sqrt{3}} \mathbf{j}+\sqrt{\frac{3}{8}} \mathbf{k}\right)$. Its norm is therefore given 
64 Comparative Analysis of Nitrides Band Structures Calculated by the Empirical Pseudopotential Method

Table 5. Pseudopotential form factors (in Ry) of wurtzite GaN, AlN and InN used in the calculation. The values of $|\mathbf{G}|^{2}$ are given in units of $(2 \pi / a)^{2}$.

\begin{tabular}{|c|c|c|c|c|c|c|c|c|c|c|c|c|c|}
\hline$|\mathbf{G}|^{2}$ & & $1 \frac{1}{3}$ & $1 \frac{1}{2}$ & $1 \frac{17}{24}$ & $2 \frac{5}{6}$ & 4 & $4 \frac{17}{24}$ & $5 \frac{1}{3}$ & $5 \frac{1}{2}$ & $5 \frac{17}{24}$ & 6 & $6 \frac{5}{6}$ & $7 \frac{1}{3}$ \\
\hline \multirow[t]{2}{*}{ GaN } & S & -0.348 & -0.371 & -0.280 & -0.053 & 0.059 & 0.083 & 0.056 & 0.066 & 0.067 & 0 & 0.021 & 0 \\
\hline & $\mathrm{V}_{\mathrm{G}}^{\mathrm{a}}$ & 0 & 0.276 & 0.225 & 0.110 & 0 & 0.051 & 0 & 0.036 & 0.033 & 0.0065 & 0.005 & 0.002 \\
\hline \multirow[t]{2}{*}{ AlN } & $\mathbf{V}_{\mathbf{G}}^{\mathrm{S}}$ & -0.363 & -0.321 & -0.130 & -0.153 & 0.034 & 0.187 & 0.076 & 0.066 & 0.058 & 0 & 0.121 & 0 \\
\hline & $V_{G}^{a}$ & 0 & 0.476 & 0.125 & 0.310 & 0 & -0.051 & 0 & 0.034 & 0.033 & 0.012 & 0.005 & -0.013 \\
\hline \multirow[t]{2}{*}{ InN } & $\mathbf{V}_{\mathbf{G}}^{\mathrm{S}}$ & -0.288 & -0.255 & -0.217 & -0.010 & 0.044 & 0.056 & 0.025 & 0.015 & 0.036 & 0 & -0.006 & 0 \\
\hline & $\mathrm{V}_{\mathrm{G}}^{\mathrm{a}}$ & 0 & 0.251 & 0.274 & 0.109 & 0 & -0.032 & 0 & 0.029 & 0.0205 & 0.0125 & 0.016 & -0.066 \\
\hline
\end{tabular}

Table 6. Eigenenergies (in eV and with respect to $\Gamma_{6}^{v}$ ) of wurtzite GaN, AlN and InN. Our calculations are evidenced by the asterisk. All other values have been taken from the literature.

\begin{tabular}{|c|c|c|c|}
\hline & $\overline{\mathrm{GaN}}$ & AlN & InN \\
\hline $\mathbf{K}_{1}^{\mathbf{v}}$ & $-5.43^{*},[16],-5.37^{[44]},-5.47^{[56]},-5.6(-6.1)^{[28]}$ & $-4.66^{*},-4.9(-5.6)^{[28]}$ & $-5.00^{*},-5.1^{[24]}$ \\
\hline$K_{3}^{v}$ & $\begin{array}{l}-5.26^{*},-5.25^{[16]}, \quad-5.29^{[44]}, \quad-5.24^{[56]} \\
-5.5(-6.1)^{[28]}\end{array}$ & $-4.52^{*},-3.9(-4.5)^{[28]}$ & $-4.93^{*},-4.9^{[24]}$ \\
\hline $\mathbf{K}_{2}^{\mathrm{v}}$ & $\begin{array}{l}-3.37^{*}, \quad-3.44^{[16]} \\
-3.0(-3.2)^{[28]}\end{array}$ & $-2.90^{*},-2.5(-3.0)^{[28]}$ & $-2.82^{*},-2.7^{[24]}$ \\
\hline$K_{3}^{v}$ & $\begin{array}{l}-2.92^{*}, \quad-2.77^{[16]} \\
-3.2(-3.5)^{[28]}\end{array}$ & $-2.51^{*},-2.5(-2.9)^{[28]}$ & $-2.57^{*},-2.6^{[24]}$ \\
\hline$K_{2}^{c}$ & $\begin{array}{l}6.35^{*},[57], 6.70^{[16]}, 4.93^{[44]}, 7.59^{[58]}, 4.9(6.6)^{[28]} \\
6.6004^{[17]}, 5.74(6.97)^{[56]}, 6.97^{[59]}\end{array}$ & $\begin{array}{l}7.94^{*}, 6.43^{[58]}, 6.29^{[57]}, \quad 7.3^{[59]} \\
7.0908^{[17]}, 4.8(6.7)^{[28]}\end{array}$ & $5.18^{*}$ \\
\hline $\mathbf{M}_{1}^{\mathrm{v}}$ & $\begin{array}{l}-6.55^{*}, \quad-6.52^{[16]}, \quad-6.56^{[44]} \\
-6.8(-7.4)^{[28]}\end{array}$ & $-5.63^{*},-5.6(-6.3)^{[28]}$ & $-5.46^{*},-6.0^{[24]}$ \\
\hline$M_{3}^{v}$ & $\begin{array}{l}-5.61^{*},-5.59^{[16]} \\
-5.6(-6.1)^{[28]}\end{array}$ & $-4.82^{*},-4.3(-4.9)^{[28]}$ & $-4.65^{*},-5.0^{[24]}$ \\
\hline$M_{1}^{v}$ & $\begin{array}{l}-4.09^{*}, \quad-3.88^{[16]} \\
-4.4(-4.9)^{[28]}\end{array}$ & $-3.52^{*},-3.4(-4.0)^{[28]}$ & $-4.29^{*},-4.0^{[24]}$ \\
\hline $\mathbf{M}_{2}^{\mathrm{v}}$ & $-2.79^{*},[16],-2.57^{[44]},-2.75^{[56]},-2.8(-3.1)^{[28]}$ & $-2.40^{*},-2.2(-2.6)^{[28]}$ & $-2.68^{*},-2.6^{[24]}$ \\
\hline$M_{3}^{v}$ & 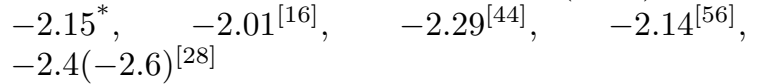 & $-1.85^{*},-1.8(-2.1)^{[28]}$ & $-2.41^{*},-2.0^{[24]}$ \\
\hline$M_{4}^{v}$ & $\begin{array}{l}-1.09^{*}, \quad-1.12^{[16]} \\
-1.0(-1.1)^{[28]}\end{array}$ & $-0.94^{*},-0.7(-0.9)^{[28]}$ & $-0.91^{*},-1.0^{[24]}$ \\
\hline$M_{1}^{c}$ & $\begin{array}{l}5.43^{*}, \quad 5.49^{[16]}, \quad 6.5777^{[17]}, \quad 5.02^{[44]}, \quad 5.94^{[57]}, \\
5.22(6.45)^{[56]}, 5.1(6.5)^{[28]}, 7.35^{[58]}, 6.45^{[59]}\end{array}$ & $\begin{array}{l}7.39^{*}, 7.03^{[58]}, 6.82^{[57]}, 7.62^{[59]} \\
7.7826^{[17]}, 5.5(7.4)^{[28]}\end{array}$ & $3.52^{*}$ \\
\hline $\mathbf{A}_{1,3}^{\mathbf{V}}$ & $\begin{array}{l}-4.18^{*}, \quad-3.97^{[16]} \\
-4.1(-4.6)^{[28]}\end{array}$ & $-3.64^{*},-3.4(-3.9)^{[28]}$ & $-4.30^{*},-3.8^{[24]}$ \\
\hline$A_{5,6}^{v}$ & $-0.55^{*,[16]},-0.49^{[44]},-0.52^{[60]},-0.5(-0.6)^{[28]}$ & $-0.49^{*},-0.6(-0.7)^{[28]}$ & $-0.56^{*},-0.5^{[24]}$ \\
\hline$A_{1,3}^{c}$ & 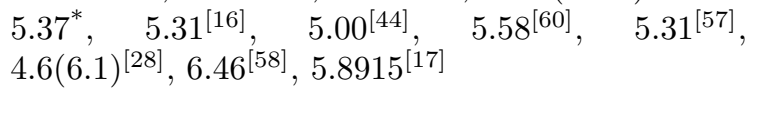 & $\begin{array}{lrr}7.35^{*}, & 7.67^{[58]}, & 7.78^{[57]} \\
8.1610^{[17]}, & 8.13^{[61]}, & 9.06^{[62,63]} \\
6.1(8.3)^{[28]} & & \end{array}$ & $3.39^{*}$ \\
\hline $\mathbf{H}_{3}^{\mathbf{v}}$ & $-5.98^{*},[60],-5.93^{[16]},-6.22^{[44]},-6.4(-7.1)^{[28]}$ & $-5.14^{*},-5.0(-5.6)^{[28]}$ & $-5.34^{*},-5.6^{[24]}$ \\
\hline $\mathbf{H}_{1,2}^{\mathbf{v}}$ & $\begin{array}{l}-4.55^{*},-4.54^{[16]}, \quad-4.35^{[44]}, \quad-3.85^{[60]}, \\
-4.6(-4.9)^{[28]}\end{array}$ & $-3.92^{*},-4.0(-4.6)^{[28]}$ & $-3.67^{*},-3.8^{[24]}$ \\
\hline $\mathbf{H}_{3}^{\mathbf{v}}$ & $\begin{array}{l}-1.43^{*}, \quad-1.40^{[16]}, \quad-1.35^{[44]} \\
-1.5(-1.6)^{[28]}\end{array}$ & $-1.22^{*},-0.8(-1.0)^{[28]}$ & $-1.78^{*},-1.5^{[24]}$ \\
\hline $\mathbf{H}_{3}^{\mathrm{c}}$ & $\begin{array}{l}7.62^{*}, \quad 7.89^{[16]}, \quad 6.62^{[44]} \\
6.6(8.3)^{[28]}, 8.5073^{[17]}\end{array}$ & $\begin{array}{llr}8.71^{*}, & 9.19^{[58]}, & 8.56^{[57]} \\
9.6651^{[17]}, & 10.38^{[61]}, & 9.96^{[62,63]} \\
7.2(9.5)^{[28]} & \end{array}$ & $4.12^{*}$ \\
\hline $\mathbf{L}_{1,3}^{\mathbf{v}}$ & $\begin{array}{l}-6.60^{*},-6.57^{[16]} \\
-7.0(-7.6)^{[28]}\end{array}$ & $-5.70^{*},-5.7(-6.5)^{\lfloor 28]}$ & $-5.72^{*},-6.0^{[24]}$ \\
\hline $\mathrm{L}_{2,4}^{\mathrm{v}}$ & $\begin{array}{l}-2.05^{*}, \quad-2.06^{[16]} \\
-2.0(-2.2)^{[28]}\end{array}$ & $-1.79^{*},-1.5(-1.8)^{[28]}$ & $-2.07^{*},-1.9^{[24]}$ \\
\hline $\mathbf{L}_{1,3}^{\mathbf{v}}$ & $\begin{array}{l}-2.00^{*}, \quad-1.95^{[16]} \\
-2.0(-2.2)^{[28]}\end{array}$ & $-1.72^{*},-1.4(-1.7)^{[28]}$ & $-1.73^{*},-1.8^{[24]}$ \\
\hline $\mathbf{L}_{1,3}^{\mathbf{c}}$ & $\begin{array}{lll}5.52^{*}, & 5.66^{[16]}, & 4.54^{[44]}, \\
4.4(6.0)^{[28]}, 6.71^{[58]}, 5.9506^{[17]} & \end{array}$ & $\begin{array}{l}7.44^{*}, \quad 7.1549^{[17]}, \quad 6.64^{[58]} \\
7.82^{[63,62]}, 6.6^{[57]}, 5.0(6.9)^{[28]}\end{array}$ & $3.04^{*}$ \\
\hline
\end{tabular}


by:

$$
|\mathbf{g}|^{2}=\left(\frac{2 \pi}{a}\right)^{2}\left[\frac{4}{3}\left(l^{2}+m^{2}-m l\right)+\frac{3}{8} n^{2}\right]
$$

Therefore, the reciprocal lattice vectors in increasing order of the modulus are in units of $(2 \pi / a)$ :

- $\mathbf{g}_{0} \equiv(0,0,0)$

- $\mathbf{g}_{\frac{3}{8}} \equiv(0,0, \pm 1)$

- $\mathbf{g}_{1+\frac{1}{3}} \equiv(-1,-1,0),(-1,0,0),(0,-1,0),(0,1,0)$, $(1,0,0),(1,1,0)$

- $\mathbf{g}_{1+\frac{1}{2}} \equiv(0,0, \pm 2)$

- $\mathbf{g}_{1+\frac{17}{24}} \equiv( \pm 1,0, \pm 1),(0, \pm 1, \pm 1),(-1,-1,-1)$, $(1,1,1),(-1,-1,1),(1,1,-1)$

- $\mathbf{g}_{2+\frac{5}{6}} \equiv(0, \pm 1, \pm 2), \quad( \pm 1,0, \pm 2), \quad(-1,-1,-2)$, $(1,1,2),(-1,-1,2),(1,1,-2)$

- $\mathbf{g}_{\mathbf{3}+\frac{\mathbf{3}}{\mathbf{8}}} \equiv(0,0, \pm 3)$

- $\mathbf{g}_{4} \equiv(1,2,0),(-1,-2,0),(2,1,0), \quad(-2,-1,0)$, $(-1,1,0),(1,-1,0)$

- $\mathbf{g}_{4+\frac{3}{8}} \equiv(1,-1, \pm 1), \quad(-1,1, \pm 1), \quad(1,2, \pm 1)$, $(-1,-2, \pm 1),(2,1, \pm 1),(-2,-1, \pm 1)$

- $\mathbf{g}_{4+\frac{17}{24}} \equiv(0, \pm 1, \pm 3),( \pm 1,0, \pm 3), \quad(-1,-1,-3)$, $(1,1,3),(-1,-1,3),(1,1,-3)$

- $\mathbf{g}_{\mathbf{5}+\frac{1}{3}} \equiv( \pm 2,0,0),(0, \pm 2,0),(2,2,0),(2,2,0)$

- $\mathbf{g}_{5+\frac{1}{2}} \equiv(1,-1, \pm 2), \quad(-1,1, \pm 2), \quad(2,1, \pm 2)$, $(1,2, \pm 2),(-2,-1, \pm 2),(-1,-2, \pm 2)$

- $\mathbf{g}_{5+\frac{17}{24}} \equiv( \pm 2,0, \pm 1), \quad(0, \pm 2, \pm 1)$, $(-2,-2,1),(2,2,-1),(-2,-2,-1)$

- $\mathbf{g}_{6} \equiv(0,0, \pm 4)$

- $\mathbf{g}_{6+\frac{5}{6}} \equiv( \pm 0, \pm 2, \pm 2), \quad( \pm 2, \pm 0, \pm 2)$, $(-2,-2,2),(2,2,-2),(-2,-2,-2)$

$\mathbf{g}_{7+\frac{1}{3}} \equiv( \pm 1, \pm 0, \pm 4),( \pm 0, \pm 1, \pm 4),(-1,-1,-3)$, $(1,1,3),(-1,-1,3),(1,1,-3)$

- $\mathbf{g}_{7+\frac{3}{8}} \equiv(-1,1, \pm 3), \quad(1,-1, \pm 3), \quad(2,1, \pm 3)$, $(1,2, \pm 3),(-2,-1, \pm 3),(-1,-2, \pm 3) \ldots$

We neglect the pseudopotential form factors corresponding to $g_{0}$ and to $\mathbf{g}^{2}>\left(7+\frac{1}{3}\right)\left(\frac{2 \pi}{a}\right)^{2}$. Besides, we can easily verify that the term $\cos \left[2 \pi\left(\frac{\Delta l}{6}+\frac{\Delta m}{6}+\frac{\Delta n}{4}\right)\right]$ corresponding to the groups of vectors $\mathbf{g}_{\frac{3}{8}}, \mathbf{g}_{\mathbf{3}+\frac{3}{8}}$ and $\mathbf{g}_{\mathbf{4}+\frac{3}{8}}$ is always null. This is the reason why the symmetrical $\left(S^{s}(|\boldsymbol{\Delta} \mathbf{g}|)\right)$ and antisymmetrical $\left(S^{a}(|\boldsymbol{\Delta} \mathbf{g}|)\right)$ structure factors for these groups of vectors are always null.

Thus, only 12 groups of vectors remain for the calculation $\left(g_{1+\frac{1}{3}}, g_{1+\frac{1}{2}}, g_{1+\frac{17}{24}}, g_{2+\frac{5}{6}}, g_{4}, g_{4+\frac{17}{24}}, g_{5+\frac{1}{3}}\right.$, $\left.\mathbf{g}_{5+\frac{1}{2}}, \mathbf{g}_{5+\frac{17}{24}}, \mathbf{g}_{\mathbf{6}}, \mathbf{g}_{\mathbf{6}+\frac{5}{6}}, \mathbf{g}_{\mathbf{7}+\frac{1}{3}}\right)$, which means 24 pseudopotential form factors: 12 symmetrical and 12 antisymmetrical. In Table 5 are given the pseudopotential form factors for wurtzite $\mathrm{GaN}$ and $\mathrm{InN}$ that we recommend and that we used in our calculations. The values of the lattice constants $a$ and $c$ that we used in the calculations are: $a=0.3189$ and $c=0.5185 \mathrm{~nm}$ for $\mathrm{GaN}$
[16, 64], $a=0.3110$ and $c=0.4980 \mathrm{~nm}[65,15]$ for AlN and $a=0.354$ and $c=0.570 \mathrm{~nm}[16,64]$ for $\operatorname{InN}$.

Therefore, the diagonal elements of the matrix $\mathrm{H}$ are given by:

$$
H_{i, i}=\left(\frac{\hbar^{2}}{2 m}\right)\left|\mathbf{k}+\mathbf{g}_{\mathbf{i}}\right|^{2}
$$

and the non-diagonal elements are given by:

$$
\begin{aligned}
H_{i, j}= & V_{s} \cos \left(\frac{\Delta l}{6}+\frac{\Delta m}{6}+\frac{\Delta n}{4}\right) \cos (\pi \Delta n u) \\
& +i V_{a} \cos \left(\frac{\Delta l}{6}+\frac{\Delta m}{6}+\frac{\Delta n}{4}\right) \sin (\pi u \Delta n)(14)
\end{aligned}
$$

As in the zinc blende structure, we have to choose a maximum value to limit the norm, given by (12), of the reciprocal vectors that are taken into consideration in the calculation. In our case, we have verified that the condition $|\mathbf{g}|^{2}<8\left(\frac{2 \pi}{a}\right)^{2}$ (corresponding to 135 vectors) provides sufficiently accurate results. Using (13) and (14), we can calculate all the elements of the Hamiltonian matrix whose diagonalization gives the eigenvalues of the electronic states corresponding to the vector $\mathbf{k}$ of the Brillouin zone.

\section{$3.3 \quad$ Results}

In figures 8, 9 and 10, the energetic band structures obtained by our pseudopotential calculations are represented for wurtzite GaN, AlN and InN respectively, along the high-symmetry points $\Gamma, K, M, A, H$ and $L$ of the Brillouin zone.

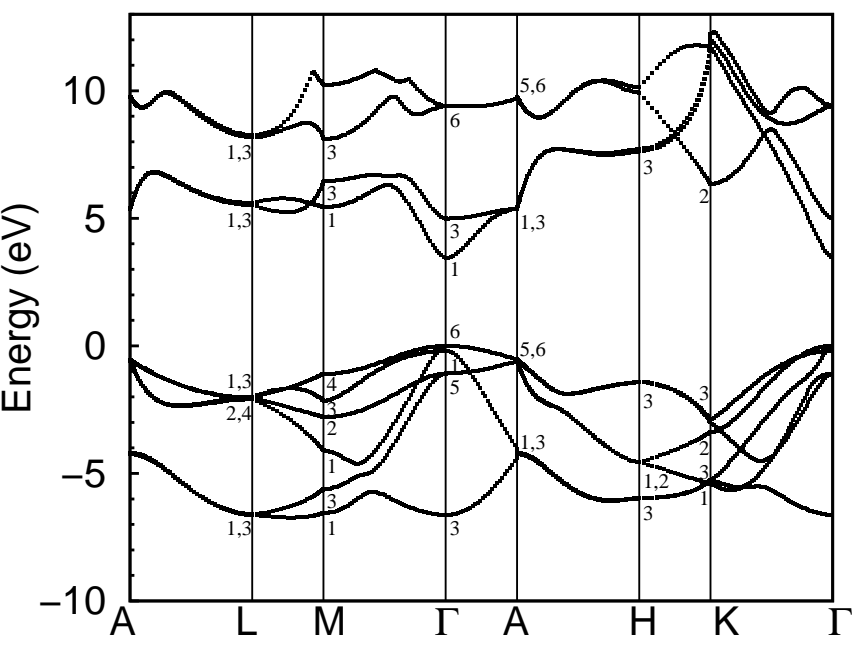

Figure 8. Band structure of wurtzite GaN obtained by our pseudopotential calculations.

From a qualitative point of view, the overall shape of the valence and conduction bands of wurtzite GaN and/or AlN is similar to that obtained by the calculations performed by Yeo et al. [16], Fritsch et al. [18], Deibuk et al. [68], Al-Douri et al. [37], Goano et al. [17], Foley et al. [19], Christensen et al. [57], Rubio et al. [28], Pugh et al. [15], Wang et al. [38], Rezaei et al. [66]. The same occurs for wurtzite InN whose overall shape of the valence and/or conduction bands is similar to the reexamined band structures of Fritsch et al. [23] and King et al. [24].

The obtained energy eigenvalues are given in Table 6 for the high-symmetry points $K, M, A, H$ and $L$. All 
66 Comparative Analysis of Nitrides Band Structures Calculated by the Empirical Pseudopotential Method

Table 7. Energy spacings, with respect to $\Gamma_{6}^{v}$, of wurtzite GaN, AlN and InN for the high-symmetry points $\Gamma, L, M, H$ and $K$. Our calculations are evidenced by the asterisk, while all other values have been taken from the literature. All values are in $\mathrm{eV}$.

\begin{tabular}{|c|c|c|c|}
\hline & GaN & AlN & InN \\
\hline$\Gamma_{1}^{\mathrm{c}}-\Gamma_{6}^{\mathrm{V}}$ & 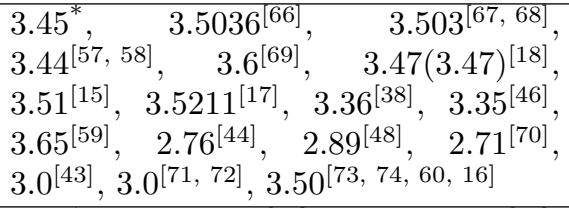 & $\begin{array}{lrr}6.20^{*}, & 6.28^{[75,63,59]}, & 6.2196^{[66]}, \\
6.24^{[15]}, & 6.2^{[61,62,63]}, & 5.8369^{[17]}, \\
6.11(6.11) & \text { citefritsch }, \quad 6.2-6.3^{[74]}, \\
4.84^{[58]}, & 6.05^{[57]}, \quad 5.84^{[17]}, & 6.21^{[68]}, \\
6.20^{[76]}, 6.29^{[77]}, 4.64^{[70]}\end{array}$ & $\begin{array}{l}0.70^{*} \\
0.824(0.790)^{[23]} \\
0.8^{[20,22]}, 0.7^{[21]}\end{array}$ \\
\hline$\Gamma_{6}^{\mathrm{v}}-\Gamma_{3}^{\mathrm{v}}$ & $\begin{array}{l}6.63^{*}, \quad 6.816^{[66]}, \quad 8.0135^{[17]}, \\
6.94(6.97)^{[18]}, 7^{[78]}, 6.8^{[16]}, 6.78^{[60]}, \\
7.18^{[44]}, 7.21^{[79]}, 7.0^{[72]}\end{array}$ & $\begin{array}{l}6.70^{*}, \quad 6.2136^{[66]}, \quad 6^{[80]}, \quad 7.0061^{[17]}, \\
6.41(6.44)^{[18]}\end{array}$ & $\begin{array}{l}6.33^{*} \\
6.024(6.154)^{[23]} \\
6.10^{[81]}\end{array}$ \\
\hline$\Gamma_{5}^{\mathrm{v}}-\Gamma_{6}^{\mathrm{v}}$ & $1.08^{*}, 1.00(1.00)^{[18]}, 1.00^{[72]}$ & $0.92^{*}, 1.03(1.04)^{[18]}$ & $\begin{array}{l}1.03^{*} \\
1.022(1.037)^{[23]}\end{array}$ \\
\hline$\Gamma_{6}^{\mathrm{v}}-\Gamma_{3}^{\mathrm{c}}$ & $\begin{array}{l}4.99^{*}, \quad 4.97^{[16]}, \quad 4.68^{[60]}, \quad 4.76^{[44]}, \\
5.8735^{[17]}\end{array}$ & $7.92^{*}, 8.2907^{[17]}$ & $2.81^{*}$ \\
\hline$\Gamma_{5}^{\mathrm{v}}-\Gamma_{3}^{\mathrm{c}}$ & $\begin{array}{l}6.07^{*}, 5.96(5.96)^{[18]}, 5.28^{[68]}, 5.3^{[69]}, \\
5.9^{[72]}\end{array}$ & $\begin{array}{l}8.84^{*}, 8.94(8.95)^{[18]}, 8.63^{[68]}, 8.92^{[76]}, \\
8.02^{[77]}\end{array}$ & $\begin{array}{l}3.84^{*} \\
3.581(3.602)^{[23]}\end{array}$ \\
\hline$\Gamma_{5}^{\mathrm{v}}-\Gamma_{6}^{\mathrm{c}}$ & $10.48^{*}, 10.74(10.74)^{[18]}, 11.1^{[72]}$ & $13.03^{*}, 12.97(12.99)^{[18]}, 14.00^{[77]}$ & $\begin{array}{l}10.15^{*} \\
9.822(9.859)^{[23]}\end{array}$ \\
\hline $\mathbf{L}_{2,4}^{\mathbf{v}}-\mathbf{L}_{1,3}^{\mathrm{c}}$ & $\begin{array}{llll}7.57^{*}, & 7.34^{[38]}, & 7.29^{[69]}, & 7.24^{[60]}, \\
7.57^{[16]}, 7.05^{[70]} & & \end{array}$ & $9.23^{*}$ & $\begin{array}{l}5.11^{*} \\
5.216(5.245)^{[23]} \\
5.41^{[81]}\end{array}$ \\
\hline $\mathbf{M}_{4}^{\mathrm{v}}-\mathrm{M}_{1}^{\mathrm{c}}$ & $\begin{array}{l}6.52^{*}, 6.60^{[38]}, 6.07(6.07)^{[18]}, 7.0^{[46]}, \\
7.05^{[60]}, 6.61^{[16]}, 6.7^{[70]}\end{array}$ & $8.33^{*}, 7.87(7.88)^{[18]}$ & $\begin{array}{l}4.43^{*} \\
4.522(4.556)^{[23]}\end{array}$ \\
\hline $\mathbf{M}_{4}^{\mathrm{v}}-\mathrm{M}_{3}^{\mathrm{c}}$ & $\begin{array}{l}7.54^{*}, 7.47^{[38]}, 7.68(7.68)^{[18]}, 7.3^{[60]} \\
7.69^{[16]}, 7.25^{[70]}, 6.8^{[68]}, 7.0-7.1^{[69]}\end{array}$ & $8.94^{*}, 8.83(8.81)^{[18]}, 9.7^{[68]}$ & $\begin{array}{l}5.12^{*} \\
5.101(5.134)^{[23]}\end{array}$ \\
\hline $\mathrm{M}_{2}^{\mathrm{v}}-\mathrm{M}_{1}^{\mathrm{c}}$ & $\begin{array}{l}8.22^{*}, 8.35^{[38]}, 7.9^{[46]}, 8.5^{[60]}, 8.26^{[16]} \\
7.13^{[68]}, 7.0-7.1^{[69]}, 7.67(7.67)^{[18]}\end{array}$ & $\begin{array}{l}9.79^{*}, \quad 10.24^{[68]} \\
9.54(9.56)^{[18]}\end{array}$ & $\begin{array}{l}6.20^{*} \\
6.090(6.142)^{[23]} \\
6.10^{[81]}\end{array}$ \\
\hline $\mathbf{H}_{3}^{\mathbf{v}}-\mathbf{H}_{3}^{\mathrm{c}}$ & $\begin{array}{l}9.05^{*}, 8.04^{[38]}, 8.4^{[60]}, 8.07(8.06)^{[18]}, \\
9.0^{[16]}\end{array}$ & $9.93^{*}, 10.91(10.10)^{[18]}, 10.39^{[77]}$ & $\begin{array}{l}5.90^{*} \\
5.892(5.883)^{[23]} \\
6.10^{[81]}\end{array}$ \\
\hline $\mathbf{K}_{2}^{\mathrm{v}}-\mathbf{K}_{2}^{\mathrm{c}}$ & $\begin{array}{l}9.72^{*}, 9.12^{[38]}, 8.68(8.68)^{[18]}, 9.0^{[46]} \\
9.45^{[60]}, 10.1^{[16]}, 9.82^{[56]}, 8.3^{[72]}\end{array}$ & $10.84^{*}, 9.57(9.59)^{[18]}$ & $\begin{array}{l}8.00^{*} \\
8.028(8.141)^{[23]}\end{array}$ \\
\hline $\mathbf{K}_{3}^{\mathrm{v}}-\mathbf{K}_{2}^{\mathrm{c}}$ & $\begin{array}{l}9.27^{*}, 9.08^{[38]}, 8.55(8.54)^{[18]}, 9.05^{[60]} \\
9.43^{[16]}, 8.29^{[68]}, 8.3-8.7^{[69]}, 7.9^{[72]}\end{array}$ & $9.43(9.43)^{[18]}$ & $\begin{array}{l}7.75^{*} \\
7.617(7.550)^{[23]}\end{array}$ \\
\hline
\end{tabular}


Table 8. Effective masses of wurtzite GaN, AlN and InN. Our calculations are evidenced by the asterisk, while all other values have been taken from the literature. All values are in units of the free electron mass.

\begin{tabular}{|c|c|c|c|}
\hline & $\overline{\mathrm{GaN}}$ & AlN & InN \\
\hline $\mathbf{m}_{\mathrm{e}}^{\|}$ & $\begin{array}{lcc}0.1712^{*}, \quad 0.16^{[15]}, & 0.18^{[82]}, \quad 0.20^{[42,48]}, \\
0.20(0.20)^{[79]}, & 0.19(0.19)^{[16,83]}, \\
0.138(0.138)^{[18]}, & 0.14(0.14)^{[84]}, & 0.2^{[85]}, \\
0.1846^{[17]}, 0.189^{[60]}, & 0.27^{[70]}, 0.197^{[86]}\end{array}$ & $\begin{array}{l}0.3006^{*}, 0.232(0.231)^{[18]} \\
0.33(0.33)^{[79]} \\
0.24(0.24)^{[84]}\end{array}$ & $\begin{array}{l}0.1076^{*} \\
0.069(0.072)^{[23]} \\
0.07^{[52]}\end{array}$ \\
\hline $\mathbf{m}_{\mathbf{e}}^{\perp}$ & $\begin{array}{lll}0.1253^{*}, & 0.12^{[15]}, \quad 0.20^{[42]}, & 0.2^{[85]} \\
0.17(0.17)^{[16]}, & 0.151(0.151)^{[18]}, & 0.18(0.18)^{[79]} \\
0.15(0.15)^{[84}, & 0.2283^{[17]}, 0.221^{[60]}, & 0.33^{[70]}, \\
0.22^{[48]}, 0.198^{[86]}, 0.23(0.19)^{[83]} & \end{array}$ & $\begin{array}{l}0.2351^{*}, 0.242(0.242)^{[18]} \\
0.25(0.25)^{[79]} \\
0.25(0.25)^{[84]}\end{array}$ & $\begin{array}{l}0.0767^{*} \\
0.069(0.068)^{[23]} \\
0.07^{[52]}\end{array}$ \\
\hline $\mathbf{m}_{\mathrm{hh}}^{\|}=\mathbf{m}_{\mathrm{lh}}^{\|}$ & $\begin{array}{l}1.9299^{*}, \quad 2.00(1.96)^{[83]}, \\
2.007(2.000)^{[18]}, \quad 1.89^{[87]}, \\
1.76(1.10)^{[79]}, 1.76(1.96)^{[16]}, 1.27^{[15]}, 2.12^{[82]}\end{array}$ & $\begin{array}{l}2.3595^{*}, 2.382(2.370)^{[18]} \\
3.53(3.68)^{[79]} \\
1.869(1.949)^{[84]} \\
3.53(4.41)^{[83]}\end{array}$ & $\begin{array}{l}1.5564^{*} \\
2.631(2.566)^{[23]} \\
1.563^{[52]}\end{array}$ \\
\hline $\mathbf{m}_{\mathrm{ch}}^{\|}$ & 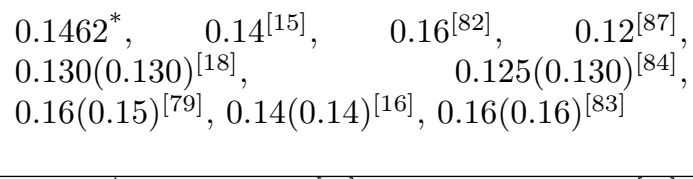 & $\begin{array}{l}0.1788^{*}, 0.209(0.209)^{[18]} \\
0.25(0.25)^{[79]} \\
0.26(0.27)^{[83]} \\
0.212(0.229)^{[84]}\end{array}$ & $\begin{array}{l}0.1506^{*} \\
0.080(0.071)^{[23]} \\
0.122^{[52]}\end{array}$ \\
\hline $\mathbf{m}_{\mathrm{hh}}^{\perp}$ & 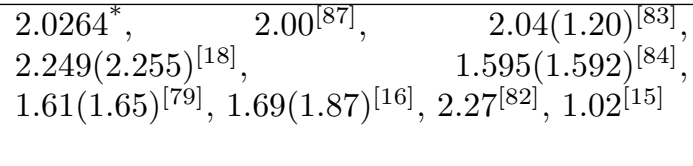 & $\begin{array}{l}2.4775^{*}, 3.040(3.058)^{[18]} \\
10.42(6.33)^{[79]} \\
2.421(2.584)^{[84]} \\
11.14(2.18)^{[83]}\end{array}$ & $\begin{array}{l}2.1638^{*} \\
2.889(2.967)^{[23]} \\
1.250^{[52]}\end{array}$ \\
\hline $\mathbf{m}_{\mathrm{lh}}^{\perp}$ & $\begin{array}{lc}0.1425^{*}, \quad 0.15^{[87]}, & 0.14(0.15)^{[79]} \\
0.261(0.191)^{[18]}, & 0.236(0.299)^{[84]} \\
0.14(0.14)^{[16]}, 0.18(0.16)^{[83]}, & 0.11^{[15]}, 0.20^{[82]}\end{array}$ & $\begin{array}{l}0.1743^{*}, 0.287(0.285)^{[18]} \\
0.24(0.25)^{[79]} \\
0.252(0.350)^{[84]} \\
0.33(0.29)^{[83]}\end{array}$ & $\begin{array}{l}0.1252^{*} \\
0.107(0.071)^{[23]} \\
0.091^{[52]}\end{array}$ \\
\hline $\mathbf{m}_{\mathrm{ch}}^{\perp}$ & 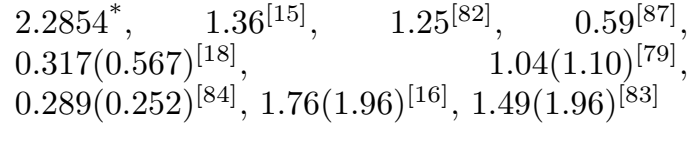 & $\begin{array}{l}2.7942^{*}, 1.157(1.204)^{[18]} \\
3.81(3.68)^{[51]} \\
1.484(0.709)^{[84]} \\
4.05(4.41)^{[83]}\end{array}$ & $\begin{array}{l}0.7906^{*} \\
1.191(1.244)^{[23]} \\
1.471^{[52]}\end{array}$ \\
\hline
\end{tabular}




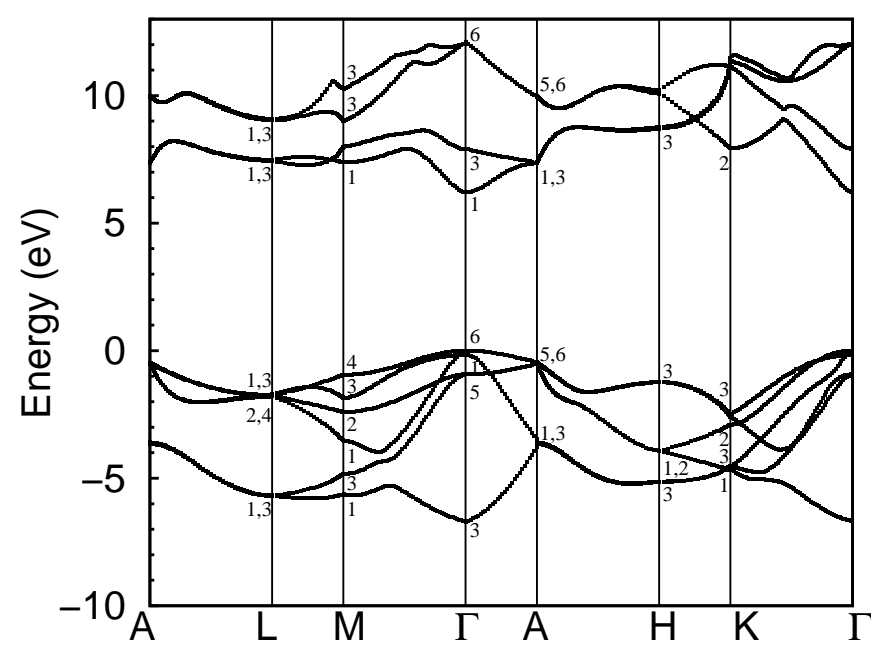

Figure 9. Band structure of wurtzite AlN obtained by our pseudopotential calculations.

eigenvalues are given with respect to the top of the valence band $\Gamma_{6}^{v}$. First we notice that, as for zinc blende InN, all results and calculations before 2002 that do not take into consideration the new corrections brought into the band structure of wurtzite InN have not been included in our comparison. In addition, the values corresponding to ref. [24] are approximate values that we have estimated from the band structure of wurtzite InN obtained by King et al. [24], since no accurate values are available in the article, but only the obtained band structure. Note that, in Table 6 and for the reference [28] (Rubio et al.), there are two proposed values for each energy eigenvalue, both calculated using the LDA method, but the value in parenthesis is the result of an additional correction that takes into consideration the electrons screening effect via the Green function (GW approach) [28]. Similarly, there are two values for the reference [56] (Lambrecht et al.): the second value in parenthesis corresponds to a correction due to the LMTO-LDA approach (linear muffin-tin orbital). An overview of the table shows a satisfactory general agreement with the results of most cited references $[17,24,28,44,56,57,58,59,60]$.

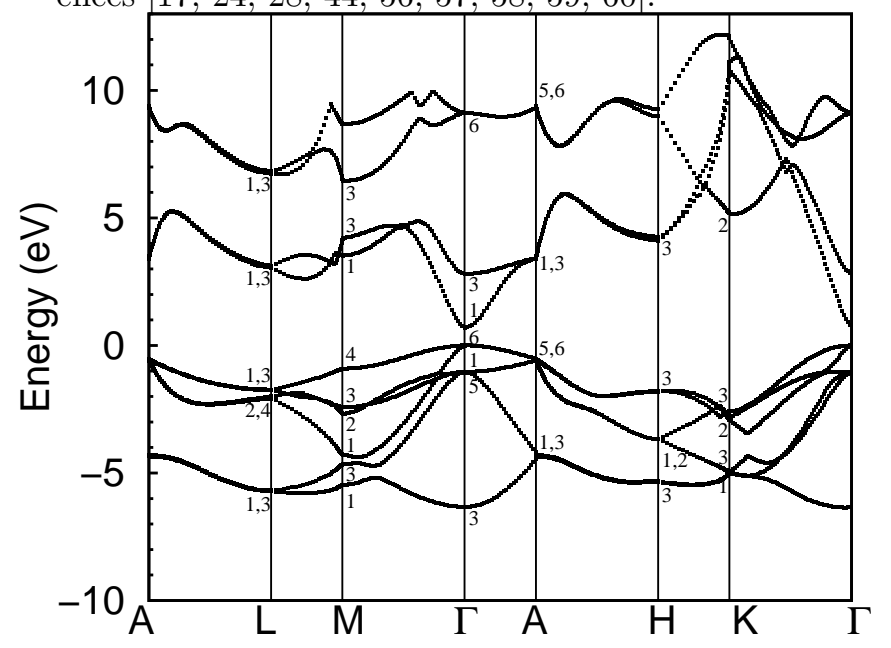

Figure 10. Band structure of wurtzite InN obtained by our pseudopotential calculations.

To ensure the accuracy of our semi-empirical results in comparison with experimental and other ab-initio and semi-empirical results, the values of energy spacings at high symmetry points $\Gamma, L, M, H$ and $K$ are given in
Table 7. In particular, the energy spacings $\Gamma_{1}^{c}-\Gamma_{6}^{v}$ and $\Gamma_{6}^{v}-\Gamma_{3}^{v}$ represent the bandgap $E_{g}$ and the valence band width respectively. Note that in Table 7 , the calculations made by Fritsch et al. [18, 23] propose two values for each energy spacing: the first corresponds to a calculation from an isotropic model of the screening effect, whereas the second value in parenthesis corresponds to an anisotropic correction of the screening effect. The validity of our semi-empirical calculations and our chosen pseudopotential form factors is well verified by the agreement of our obtained GaN and/or AlN energy spacings with other calculations, like the calculations made by Yeo et al. [16], Fritsch et al. [18], Wang et al. [38] and others. Furthermore, our chosen pseudopotential form factors are well adapted to the latest experimental and theoretical results that take into consideration the band gap corrections of wurtzite InN [20, 21, 22, 23].

A parabolic line fit is also used to determine the effective masses of the electrons for wurtzite GaN, AlN and InN. The lowermost conduction band shows slight anisotropy for $\mathbf{k}$ directions. For $\mathrm{GaN}, m_{e}^{\|}=0.1712 m_{0}$ in the $k_{z}$ direction, and $m_{e}^{\perp}=0.1253 m_{0}$ in the $k_{x} k_{y}$ plane. For $\mathrm{AlN}, m_{e}^{\|}=0.3006 m_{0}$ in the $k_{z}$ direction, and $m_{e}^{\perp}=0.2351 m_{0}$ in the $k_{x} k_{y}$ plane. For InN, $m_{e}^{\|}=0.1076 m_{0}$ in the $k_{z}$ direction, and $m_{e}^{\perp}=0.0767 m_{0}$ in the $k_{x} k_{y}$ plane. Our results along with other available data in the literature are shown in Table 8 . Note first that in Table 8, regarding the results of Fritsch et al. $[18,23]$, there are two proposed values for each of the calculated effective masses, the value in parenthesis corresponds to an additional anisotropic correction. Dudgale et al. [84] also propose two values: the first is calculated directly by the $\mathbf{k} \cdot \mathbf{p}$ method, the second by the Monte Carlo method. Similarly, there are two values proposed by Suzuki et al. [79] for each of the effective masses, the second in brackets resulting in a further correction by a $3 \mathrm{D}$ interpolation procedure within the cubic approximation. As for Yeo et al. [16] values, one comes from the parabolic approximation, the other is due to the $\mathbf{k} \cdot \mathbf{p}$ method. Finally, the second value given by Kim et al. [83] is obtained through a quasicubic model calculation. The discrepancy between the values given by the cited references does not preclude the fact that our values are consistent with several values proposed by other calculations, especially those of Ren et al. [87], but also those of Fritsch et al. $[18,23]$ and Yeo et al. [16], despite the use of different approximations and calculation methods.

\section{Comparative analysis}

In Table 9 the main characteristics and parameters that we can deduce from the energetic band structures of GaN, AlN and InN are grouped for both zinc blende and wurtzite phases. In particular, we have reported the bandgap value but also other important parameters such as the effective masses, the nonparabolicity coefficient of the $\Gamma$ valley and the valence band width. The results show that zinc blende $\mathrm{GaN}$ and InN are directgap semiconductors with the minimum of the conduction band at $\Gamma$ point, whereas zinc blende AlN has an indirect gap with the minimum of conduction band at 
Table 9. Comparison of the main parameters of GaN, AlN and InN in both zinc blende and wurtzite phases. All energetic values are in $\mathrm{eV}$. All effective masses are in units of the free electron mass $m_{0}$. The nonparabolicity coefficient is given in eV ${ }^{-1}$.

\begin{tabular}{|c|c|c|c|c|c|c|c|}
\hline & & \multicolumn{2}{|l|}{$\overline{\mathrm{GaN}}$} & \multicolumn{2}{|l|}{$\overline{\mathrm{AlN}}$} & \multicolumn{2}{|l|}{$\overline{\mathrm{InN}}$} \\
\hline & & ZB & WZ & ZB & WZ & ZB & WZ \\
\hline Direct/indirect gap & & direct & direct & indirect & direct & direct & direct \\
\hline Bandgap value & & 3.23 & 3.45 & 4.91 & 6.21 & 0.51 & 0.70 \\
\hline Valence band width & & 6.15 & 6.63 & 5.86 & 6.70 & 6.51 & 6.33 \\
\hline Effective masses & $m_{e}$ & 0.1393 & & 0.271 & & 0.1140 & \\
\hline & $m_{e}^{\|}$ & & 0.1712 & & 0.3006 & & 0.1076 \\
\hline & $m_{e}^{\perp}$ & & 0.1253 & & 0.2351 & & 0.0767 \\
\hline Nonparabolicity coefficient & & 0.229 & 0.199 & 0.090 & 0.079 & 1.539 & 1.138 \\
\hline
\end{tabular}

$X$ point. The calculated values of the bandgap $E_{g}^{\Gamma-\Gamma}$ of GaN and $\mathrm{InN}$ are 3.23 and $0.51 \mathrm{eV}$ respectively, and the bandgap $E_{g}^{\Gamma-X}$ of AlN is equal to $4.91 \mathrm{eV}$. These values are in good agreement with other experimental and theoretical results, as we have already shown in Table 3. As for wurtzite GaN, AlN and InN, they are direct-gap semiconductors with the minimum of conduction band at $\Gamma$ point. The values of our calculated bandgaps for wurtzite GaN, AlN and $\mathrm{InN}$ are 3.45, 6.20 and $0.70 \mathrm{eV}$ respectively, thus covering a wide energy range of the electromagnetic spectrum and as a consequence more potential applications. On the other hand, all studied nitrides have similar values of the valence band width $(\approx 6 \mathrm{eV})$ and AlN in both its zinc blende and wurtzite phases has a larger effective mass than GaN and InN. Finally, we have also calculated the nonparabolicity coefficient $\alpha$ in the $\Gamma$ valley of the conduction band, a very important parameter for instance in electronic devices transport simulations. It can be easily calculated using the approximation $\alpha=\left(1 / E_{g}\right)\left(1-m^{*} / m_{0}\right)^{2}$, where $E_{g}$ is the bandgap value and $m^{*}$ the electron effective mass in the $\Gamma$ valley.

\section{Conclusion}

We have presented reexamined band structures for zinc blende and wurtzite $\mathrm{InN}$ in agreement with the latest corrections brought into its bandgap values, as well as a comparative study of the electronic band structures of zinc blende and wurtzite GaN, AlN and InN using the empirical pseudopotential approach. The obtained band structures are validated by a comparison with most of the experimental data and ab-initio calculations reported in the literature. GaN and $\mathrm{InN}$ in both zinc blende and wurtzite structures, as well as wurtzite AlN have direct bandgaps, but zinc blende AlN has an indirect gap at $X$ point. All calculated bandgaps values are found to be in agreement with other experimental and theoretical results. In particular, zinc blende and wurtzite InN bandgap values are consistent with the latest measurements that clearly demonstrated that the bandgap value of $\mathrm{InN}$ is lower than $1 \mathrm{eV}$, much lower than the bandgap values of (zinc blende/wurtzite) GaN (3.23/3.45 eV) and (zinc blende/wurtzite) AlN $(4.91 / 6.20 \mathrm{eV})$. The effective mass parameters are also derived using a parabolic fit which has proven to be a reliable approximation in most cases.

For practical purposes, all the optimized symmetrical and antisymmetrical pseudopotential form factors have been tabulated to fill the lack in the literature regarding the empirical pseudopotential parameters of zinc blende and wurtzite III-nitrides. Furthermore, we have tabulated the eigenenergies at high-symmetry points, valley separation energies and bandgaps, electron and hole effective masses, and nonparabolicity coefficients, all of which are crucial parameters for microscopic numerical simulations of electronic and optoelectronic devices.

\section{REFERENCES}

[1] W Shan, J.W.III Ager, K.M. Yu, W. Walukiewicz, Haller E.E., M.C. Martin, W.R. McKinney, and W. Yang. Dependence of the fundamental band gap of $\mathrm{Al}_{x} \mathrm{Ga}_{1-x} \mathrm{~N}$ on alloy composition and pressure. $J$. Appl. Phys., 85(12):8505-, 1999.

[2] S. Nakamura, M. Senoh, S. Nagahama, N. Iwasa, T. Yamada, T. Matsushita, H. Kiyoku, and Y. Sugimoto. InGaN-based multi-quantum-well-structure laser diodes. Jpn. J. Appl. Phys., Part 2, 35:L74L76, 1996.

[3] I. Akasaki, H. Amano, S. Sota, H. Sakai, T. Tanaka, and M. Koike. Stimulated emission by current injection from an AlGaN/GaN/GaInN quantum well device. Jpn. J. Appl. Phys., Part 2, 34(11B):L1517L1519, 1995.

[4] H. Morkoc and S.N. Mohammad. High-luminosity blue and blue-green gallium nitride light-emitting diodes. Science, 267(5194):51-55, 1995.

[5] W. Orton and C. T. Foxon. Group III nitride semiconductors for short wavelength light-emitting devices. Rep. Prog. Phys., 61(1):1-75, 1998.

[6] F.A. Ponce and D.P. Bour. Nitride-based semiconductors for blue and green light-emitting devices. Nature London, 386(1):351-359, 1997.

[7] S.C. Jain, M. Willander, J. Narayan, and R. Van Overstraeten. III-nitrides: growth, characterization, and properties. J. Appl. Phys., 87(3):965-, 2000 .

[8] Y.F. Wu, B.P. Keller, S. Keller, D. Kapolnek, P. Kozodoy, S.P. Denbaars, and U.K. Mishra. Very high breakdown voltage and large transconductance realized on GaN heterojunction field effect transistors. Appl. Phys. Lett., 69(10):1438-, 1996. 
[9] P. Shiktorov, E. Starikov, V. Gružinskis, L. Varani, C. Palermo, J. Torres, and L. Chusseau. Generation of terahertz radiation assisted by optical phonons in nitride-based quantum wells and heterolayers. Semicond. Phys. Inst., Vilnius, 5(1):236-239, 2008.

[10] E. Starikov, P. Shiktorov, V. Gružinskis, L. Varani, C. Palermo, J.-F. Millithaler, and L. Reggiani. Terahertz generation in nitrides due to transit-time resonance assisted by optical phonon emission. $J$. Phys.: Condens. Matter, 20:384209-384221, 2008.

[11] L. Varani, J.C. Vaissière, E. Starikov, P. Shiktorov, V. Gružinskis, L. Reggiani, and J.H. Zhao. Monte carlo calculations of $\mathrm{THz}$ generation in nitrides. Phys. Stat. Solidi A, 190(1):247-256, 2001.

[12] S.C.P. Rodrigues, A.L. Rosa, L.M. R. Scolfaro, D. Beliaev, J.R. Leite, R. Enderlein, and J.L.A. Alves. Miniband structures and effective masses of n-type doping superlattices in GaN. Semicond. Sci. Technol., 13(9):981-988, 1998.

[13] E.A. Albanesi, W.R.L. Lambrecht, and B. Segall. Electronic structure and equilibrium properties of $\mathrm{Ga}_{x} \mathrm{Al}_{1-x} \mathrm{~N}$ alloys. Phys. Rev. B, 48(24):1784117847, 1993.

[14] H. Amano, N. Serwai, I. Akasaki, and Y. Ttovoda. Metalorganic vapor phase epitaxial growth of a high quality GaN film using an AlN fubber layer. Appl. Phys. Lett., 48(5):353-355, 1986.

[15] S.K. Pugh, D. J. Dugdale, Brand S., and Abram R.A. Electronic structure calculations on nitride semiconductors. Semicond. Sci. Technol., 14(1):2331,1999 .

[16] Y. C. Yeo, T. C. Chong, and M. F. Li. Electronic band structures and effective mass parameters of wurtzite GaN and InN. J. Appl. Phys., 83(3):1429 , 1998.

[17] M. Goano, E. Bellotti, E. Ghillino, G. Ghione, and K.F. Brennan. Band structure nonlocal pseudopotential calculation of the III-nitride wurtzite phase materials system. Part I. binary compounds GaN, AlN, and InN. J. Appl. Phys., 88(11):6467-, 2000.

[18] D. Fritsch, H. Schmidt, and M. Grundmann. Bandstructure pseudopotential calculation of zinc-blende and wurtzite AlN, GaN, and InN. Phys. Rev. B, 67(23):235205.1-235205.13, 2003.

[19] C.P. Foley and T.L. Tansley. Pseudopotential band structure of indium nitride. Phys. Rev. B, 33(2):1430-, 1986.

[20] J. Wu, W. Walukiewicz, K. M. Yu, J. W. Ager III, E. E. Haller, H. Lu, W. J. Schaff, Y. Saito, and Y. Nanishi. Unusual properties of the fundamental band gap of InN. Appl. Phys. Lett., 80(21):39673969, 2002.

[21] V. Yu. Davydov, A. A. Klochikhin, R. P. Seisyan, V. V. Emtsev, S. V. Ivanov, F. Bechstedt, J. Furthmuller, H. Harima, A. V. Mudryi, J. Aderhold,
O. Semchinova, and J. Graul. Absorption and emission of hexagonal InN. evidence of narrow fundamental band gap. Phys. Stat. Sol. (b), 229(3):R1R3, 2002.

[22] Y. Nanishi, Y. Saito, and T. Yamaguchi. RFmolecular beam epitaxy growth and properties of InN and related alloys. Jpn. J. Appl. Phys., 42(5A):2549-2559, 2003.

[23] D. Fritsch, H. Schmidt, and M. Grundmann. Band dispersion relations of zinc-blende and wurtzite InN. Phys. Rev. B, 69(8):165204.1-165204.5, 2004.

[24] P.D.C King, T.D. Veal, C.F. McConville, F. Fuchs, J. Furthmüller, F. Bechstedt, J. Schörmann, D.J. As, K. Lischka, H. Lu, and W.J. Schaff. Valence band density of states of zinc-blende and wurtzite InN from x-ray photoemission spectroscopy and ïst-principles calculations. Phys. Rev. B, 77:115213.1-115213.7, 2008.

[25] Ch. Kittel. Introduction to Solid State Physics. Wiley New York, 1996.

[26] P.Y. Yu and M. Cardona. Fundamentals of semiconductors, physics and materials properties. Springer, 1995.

[27] H. Aourag, B. Bouhafs, and M. Certier. Empirical pseudo-potential calculations in $\mathrm{GaAs}_{1-x} \mathrm{~N}_{x}$ and $\mathrm{AlAs}_{1-x} \mathrm{~N}_{x}$ ordered alloys. Phys. Status Solidi B, 201(1):117-, 1997.

[28] A. Rubio, J.L. Corkill, M.L. Cohen, E.L. Shirley, and S.G. Louie. Quasiparticle band structure of AlN and GaN. Phys. Rev. B, 48(16):11810-11816, Oct 1993.

[29] W.J. Fan, M.F. Li, T.C. Chong, and J.B. Xia. Electronic properties of zinc blende GaN, AlN and their alloys $\mathrm{Ga}_{1-x} \mathrm{Al}_{x}$ N. J. Appl. Phys., 79(1):188-194, 1996.

[30] A. Tadjer, B. Abbar, M Rezki, H. Aourag, and M. Certier. Electronic properties and strain effects in zinc blende GaN and InN. J. Phys. Chem. Solids, 60(3):419-424, 1999.

[31] F. Bechstedt and J. Furthmüller. J. Cryst. Growth, 246:135-, 2002.

[32] W. R. L. Lambrecht, B. Segall, J. Rife, W. R. Hunter, and D. K. Wickenden. UV reflectivity of GaN: theory and experiment. Phys. Rev. B, 51(19):13516-13532, May 1995.

[33] H. Aourag and M. Certier. Electronic structure of III-V nitrides semiconductors. J. Phys. Chem. Solids, 59(6-7):1145-1156, 1998.

[34] T. Lei, M. Fanciulli, R.J. Molnar, and T.D. Moustakas. Epitaxial growth of zinc blende and wurtzitic gallium nitride thin films on (001) silicon. Appl. Phys. Lett., 59(8):944-, 1991.

[35] S. Strite and H. Morkoc. GaN, AlN and InN: a review. J. Vac. Sci. Technol. B, 10(1237), 1992. 
[36] G. Steude, D.M Hoffmann, B.K. Meyer, H. Amano, and I. Akasaki. The dependence of the band gap on alloy composition in strained AlGaN on GaN. Phys. Status Solidi B, 205(1), 1998.

[37] Y. Al-Douri. Electronic and positron properties of zinc-blende structure of $\mathrm{GaN}$, AlN, and their alloy $\mathrm{Ga}_{1-x} \mathrm{Al}_{x} \mathrm{~N}$. J. Appl. Phys., 93(12):9730-9736, 2003.

[38] R. Wang, P.P. Ruden, J. Kolnik, I Oguzman, and K.F. Brennan. Dielectric properties of wurtzite and zinc blende structure gallium nitride. J. Phys. Chem. Solids, 58(6):913-918, 1997.

[39] M. Fanciulli, T. Lei, and T. D. Moustakas. Conduction-electron spin resonance in zinc-blende GaN thin films. Phys. Rev. B, 48(20):15144-15147, Nov 1993.

[40] A. Tadjer, B Abbar, M Rezki, J.P. Dufour, H. Aourag, and M. Certier. Charge density calculations for strained zinc blende GaN, InN and AlN. Mater. Chem. Phys., 62(1):75-80, 2000.

[41] A. Bhouri, F. Ben Zid, H. Mejri, A. Ben Fredj, N. Bouarissa, and M. Said. Electronic band parameters for zinc-blende $\mathrm{Al}_{1-x} \mathrm{Ga}_{x} \mathrm{~N}$. J. Phys: Condens. Matter, 14(29):7017-7026, 2002.

[42] I. Vurgaftman, J. R. Meyer, and L. R. Ram-Mohan. Band parameters for III-V compound semiconductors and their alloys. J. Appl. Phys., 89(11):5815-, 2001.

[43] B.J. Min, C.T. Chan, and K.M. Ho. First-principles total-energy calculation of gallium nitride. Phys. Rev. B, 45(3):1159-, 1992.

[44] M. Palummo, L. Bertoni, C. M. Reining, and F. Finochi. The electronic structure of gallium nitride. Physica B, 185(1-4):404-409, 1993.

[45] S. Bounab, Z. Charifi, and N. Bouarissa. Electronic and positronic properties of $\mathrm{Al}_{1-x} \mathrm{In}_{x} \mathrm{~N}$ with zinc blende structure. Physica B, 324(1-4):72-81, 2002.

[46] S. Logothetidis, J. Petalas, M. Cardona, and T.D. Moustakas. Optical properties and temperature dependence of the interband transitions of cubic and hexagonal GaN. Phys. Rev. B, 50(24):18017-18029, 1994.

[47] A.T. Meney, E.P. O'reilly, and A.R. Adams. Optical gain in wide bandgap GaN quantum well lasers. Semicond. Sci. Technol., 11(6):897-903, 1996.

[48] K. Miwa and A. Fukumoto. First-principles calculation of the structural, electronic, and vibrational properties of gallium nitride and aluminum nitride. Phys. Rev. B, 48(11):7897-7902, 1993.

[49] K. Kassali and N. Bouarissa. Pseudopotential calculations of electronic properties of $\mathrm{Ga}_{1-x} \mathrm{In}_{x} \mathrm{~N}$ alloys with zinc-blende structure. Solid-State Electron., 44(3):501-507, 2000.
[50] L. E. Ramos, L. K. Teles, L. M. R. Scolfaro, J. L. P. Castineira, A. L. Rosa, and J. R. Leite. Structural, electronic, and effective-mass properties of silicon and zinc-blende group-III nitride semiconductor compounds. Phys. Rev. B, 63(16):165210165219, 2001.

[51] M. Suzuki, T. Uenoyama, and A. Yanase. Optical gain and crystal symmetry in III-V nitride laser. Appl. Phys. Lett., 69(22):3378-, 1996.

[52] I. Vurgaftman and J.R. Meyer. Band parameters for nitrogen-containing semiconductors. J. Appl. Phys., 94(6):3675-3696, 2003.

[53] W.J. Fan, M.F. Li, T.C. Chong, and J.B. Xia. Band structure parameters of zinc blende GaN, AlN and their alloys $\mathrm{Ga}_{1-x} \mathrm{Al}_{x} \mathrm{~N}$. Solid State Commun., 97(5):381-384, 1996.

[54] Y.-K. Kuo, B.-T. Liou, S.-H. Yen, and H.-Y. Chu. Vegardâs law deviation in lattice constant and band gap bowing parameter of zinc blende $\operatorname{In}_{x} \mathrm{Ga}_{1-x} \mathrm{~N}$. Optics Communications, 237:363-369, 2004.

[55] V. Cimalla, J. Pezoldt, G. Ecke, R. Kosiba, O. Ambacher, L. Spie $\beta$, and G. Teichert. Growth of cubic InN on r-plane sapphire. Appl. Phys. Lett., 83(17):3468-, 2003.

[56] W.R.L. Lambrecht and B. Segall. Properties of Group III Nitrides. INSPEC, IEE, London, 1994.

[57] N.E. Christensen and I. Gorczyca. Optical and structural properties of III-V nitrides under pressure. Phys. Rev. B, 50(7):4397-4415, 1994.

[58] T. V. Gorkavenko, S. M. Zubkova, and L. N. Rusina. Temperature dependence of the band structure of wurtzite-type semiconductor compounds: gallium and aluminum nitrides. Semiconductors, 41(6):641-650, 2007.

[59] K. Kim, W. R. Lambrecht, and B. Segal. Elastic constants and related properties of tetrahedrally bonded BN, AlN, GaN, and InN. Phys. Rev. B, 53(24):16310-16326, 1996.

[60] M.-Z. Huang and W. Y. Ching. Minimal basis semiab initio approach to the band structures of semiconductors. J. Phys. Chem. Solids, 46(8):977-995, 1985.

[61] A. Kobayashi, O. F. Sankey, S. M. Volz, and J. D. Dow. Semiempirical tight-binding band structures of wurtzite semiconductors: AlN, CdS, CdSe, ZnS, and ZnO. Phys. Rev. B, 28(2):935-945, 1983.

[62] B. Heida and K. Hauptmanova. Phys. Stat. Solidi, 36:K95-, 1969.

[63] P.B. Perry and R.F. Rutz. The optical absorption edge of single-crystal AlN prepared by a closespaced vapor process. Appl. Phys. Lett., 33(4):319-, 1978.

[64] R.F. Davis. III-V nitrides for electronic and optoelectronic applications. Proc. IEEE, 79(5):702-712, 1991. 
[65] H. Schulz and K.H. Thiemann. Crystal structure refinement of AlN and GaN. Solid State Commun., 23(11):815-819, 1977.

[66] B. Rezaei, A. Asgari, and M. Kalafi. Electronic band structure pseudopotential calculation of wurtzite III-nitride materials. Physica B, 371(1):107-111, 2006.

[67] B. Monemar. Fundamental energy gap of GaN from photoluminescence excitation spectra. Phys. Rev. $B, 10(2): 676-681,1974$.

[68] V. G. Deibuk, A. V. Voznyi, and M. M. Sletov. Band structure and spatial charge distribution in $\mathrm{Al}_{x} \mathrm{Ga}_{1-x} \mathrm{~N} . \quad$ Semiconductors, 34(1):35-39, 2000. Translated from Fizika i Tekhnika Poluprovodnikov, Vol. 34, No. 1, 2000, pp. 36-40.

[69] S. Bloom, G. Harbecke, E. Meier, and I.B. Ortenburger. Band structure and reflectivity of GaN. Phys. Stat. Solidi B, 66(1):161-168, 1974.

[70] Y.N. Xu and W.Y. Ching. Electronic, optical, and structural properties of some wurtzite crystals. Phys. Rev. B, 48(7):4335-, 1993.

[71] S. N. Grinyaev, V. Ya Malakhov, and V. A. Chaldyshev. Calculation of the band structure of GaN and InN using the pseudopotential method. Russian Physics Journal, 29(4):311-314, 2004.

[72] G.L. Zhao, D. Bagayoko, and T.D. Williams. Local-density-approximation prediction of electronic properties of $\mathrm{GaN}, \mathrm{Si}, \mathrm{C}$, and $\mathrm{RuO}_{2}$. Phys. Rev. B, 60(3):1563-, 1990.

[73] R. Dingle, D.D. Sell, S.E. Stokowski, and M. Ilegems. Absorption, reflectance, and luminescence of GaN epitaxial layers. Phys. Rev. B, 4(4):12111218, 1971.

[74] K.H. Hellwege. Numerical data and functional relationships in science and technology (LandoltBörnstein Tables III/17a).

[75] L. Roskovcova and J. Pastrnak. The 'Urbach' absorption edge in AlN. Czech J. Phys. B, 30(5):586$591,1980$.

[76] A. P. Dmitriev, N. V. Evlakhov, and A. S. Furman. Pseudopotential calculation of the band structure of the solid solution SiC-AlN. Semiconductors, 30(1):60-67, 1996. Translated from Fizika i Tekhnika Poluprovodnikov, Vol. 35, No. 2, 2001, pp. 216-218.
[77] S. Loughin, R.H. French, W.Y. Ching, Y.N. Xu, and G.A. Slack. Electronic structure of aluminum nitride: theory and experiment. Appl. Phys. Lett., 63(9):1182-, 1993.

[78] R.W. Hunt, L. Vanzetti, T. Castro, K.M. Chen, L. Sorba, P.I. Cohen, W. Gladfelter, J. M. van Hove, J.N. Kuznia, M.A. Khan, and A. Fransiosi. Electronic structure, surface composition and longrange order in GaN. Physica B, 185(1-4):415-421, 1993.

[79] M. Suzuki, T. Uenoyama, and A. Yanase. Firstprinciples calculations of effective-mass parameters of AlN and GaN. Phys. Rev. B, 52(11):8132-8139, 1995.

[80] M. Gautier, J.P. Duraud, and C. Le Gressus. Electronic structure of an AlN film produced by ion implantation, studied by electron spectroscopy. $J$. Appl. Phys., 61(2):574-580, 1994.

[81] A. Kasic, E. Valcheva, B. Monemar, H. Lu, and L. F. Eastman. Inn dielectric function from the midinfrared to the ultraviolet range. Phys. Rev. B, 70(11):115217-, 2004.

[82] J.A. Majewski, M. Städele, and P. Vogl. Electronic structure of biaxially strained wurtzite crystals GaN, AlN, and InN. Materials Research Society Internet J. Nitride Semicond. Research, 1:30-, 1996.

[83] K Kim, W.R.L Lambrecht, B. Segall, and M. van Schilfgaarde. Effective masses and valenceband splittings in GaN and AlN. Phys. Rev. B, 56(12):7363-7375, 1997.

[84] D.J. Dudgale, S. Brand, and R.A. Abram. Direct calculation of kâp parameters for wurtzite AlN, GaN, and InN. Phys. Rev. B, 61(19):12933-12938, 2000 .

[85] B. Jogai. Effective masses of wurtzite GaN calculated from an empirical tight binding method. Solid State Commun., 107(7):345-348, 1998.

[86] T. Yang, S. Nakajima, and S. Sakai. Electronic structures of wurtzite GaN, InN and their alloy $\mathrm{Ga}_{1-x} \operatorname{In}_{x} \mathrm{~N}$ calculated by the tight-binding method. Jpn. J. Appl. Phys., Part 1, 34(11):5912-5921, 1995.

[87] G.B. Ren, Y.M. Liu, and P. Blood. Valence-band structure of wurtzite GaN including the spin-orbit interaction. Appl. Phys. Lett., 74(8):1117-, 1999. 